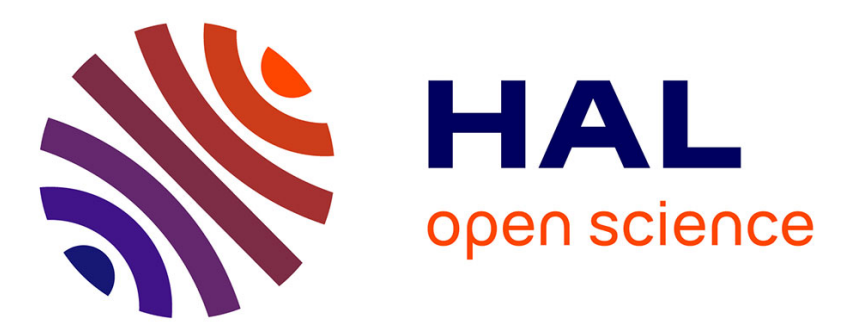

\title{
Optimal Exciting Dance for Identifying Inertial Parameters of an Anthropomorphic Structure
}

Vincent Bonnet, Philippe Fraisse, André Crosnier, Maxime Gautier, Alejandro González, Gentiane Venture

\section{- To cite this version:}

Vincent Bonnet, Philippe Fraisse, André Crosnier, Maxime Gautier, Alejandro González, et al.. Optimal Exciting Dance for Identifying Inertial Parameters of an Anthropomorphic Structure. IEEE Transactions on Robotics, 2016, 32 (4), pp.823-836. 10.1109/TRO.2016.2583062 . lirmm-01346044

\section{HAL Id: lirmm-01346044 https://hal-lirmm.ccsd.cnrs.fr/lirmm-01346044}

Submitted on 18 Jul 2016

HAL is a multi-disciplinary open access archive for the deposit and dissemination of scientific research documents, whether they are published or not. The documents may come from teaching and research institutions in France or abroad, or from public or private research centers.
L'archive ouverte pluridisciplinaire HAL, est destinée au dépôt et à la diffusion de documents scientifiques de niveau recherche, publiés ou non, émanant des établissements d'enseignement et de recherche français ou étrangers, des laboratoires publics ou privés. 


\title{
Optimal Exciting Dance for Identifying Inertial Parameters of an Anthropomorphic Structure
}

\author{
Vincent Bonnet, Philippe Fraisse, André Crosnier, Maxime Gautier, Alejandro González, and Gentiane Venture
}

\begin{abstract}
Knowledge of the mass and inertial parameters of a humanoid robot or a human being is crucial for the development of model-based control as well as for monitoring the rehabilitation process. These parameters are also important for obtaining realistic simulations in the field of motion planning and human motor control. For robots they are often provided by CAD data while averaged anthropometric tables values are often used for human subjects. The unit/subject specific inertial parameters can be identified using the external wrench caused the ground reaction. However, the identification accuracy intrinsically depends on the excitation properties of the recorded motion. In this paper, a new method for obtaining optimal excitation motions is proposed. This method is based on the identification model of legged systems and on optimization processes to generate excitation motions while handling mechanical constraints. A pragmatic decomposition of this problem, the use of a new excitation criterion and a quadratic program to identify inertial parameters are proposed. The method has been experimentally validated onto a HOAP-3 humanoid robot and with one human subject.
\end{abstract}

Index Terms-dynamics identification, human, humanoid robot, exciting motion.

\section{INTRODUCTION}

Both humanoid robots and the human body are highly nonlinear redundant systems with specific unit/subject body segment inertial parameters. For humanoids these parameters are usually obtained using CAD data. However, they do not take into account cabling, covers, glued components, embedded computers, or the several modifications that come with the use of a multipurpose robot. Good knowledge of these parameters is important when using model-based controllers to guarantee stability since the robustness and accuracy of a robot controller depends on the ability to predict dynamic behavior. Even when using balance controllers based on a simplified linear inverted pendulum, the performance of the controller depends on the standard inertial parameters (SIP) used to estimate the position of the total center of mass (CoM) [1]. The manual and individual measurement of the SIP (the mass of each segment, the 3D CoM position, and inertia matrix) appears cumbersome or impossible for such complex systems. The human SIP are mostly estimated from anthropometric tables (AT) [2] obtained from cadavers data. These tables only account for variations within a relatively small category of subjects. This is problematic when dealing with individuals presenting an atypical body mass distribution such as the elderly, infant or obese.

\section{A. State of the art in model based dynamics identification}

The last decade has seen the development of dynamic identification methods for floating base systems [3], [4], [5],
[6] inspired by the work on manipulators of Dubowsky et al. [7]. When dealing with serial manipulators joint torque measurements are often available and used. This is not the case for most anthropomorphic structures for which taking advantage of the external generalized forces and moments was been proposed [3], [6]. For humans, SIP identification was performed by having the subject mimic popular rehabilitation motions or using a visual biofeedback system capable of displaying the evolution of the identification process of each link [3]. Such systems work fine, but the time required for any given subject may vary depending on her psychological/physiological capabilities. It is possible to extract the most exciting motions for the identification from a set of random ones using the sub-regressor matrices [8]. This method requires a large database of different motions and does not ensure that all inertial parameters are excited. In order to normalize the identification process and to minimize the required time, it is preferable to use a set of motions specifically designed to excite the SIP. However, due to the high complexity of the anthropomorphic structures these optimal exciting motions are difficult to determine. When designing a set of identification motions for humanoid robots and humans the range of feasible motions, the dynamic balance, and mechanical limitations must taken into consideration. In order to cope with these constraints the literature regarding serial manipulators proposes to generate exciting motions using optimization approaches. Most of these use joint trajectory represented by Fourier Series [9], [10], [11] or B-splines [12] that minimize a criterion related to the condition number of the whole regressor [11], [13]. These methods have been applied and extended to humans [14]. A humanoid robot is intrinsically unbalanced, has a large number of SIP to identify, and has a larger number of degrees-of-freedom (DoF) to control implying a de-facto use of large-scale optimization processes. These imply convergence problems, and numerous special cases to handle (single or double support, etc). Also, the condition number of the regressor, that is the ratio of the largest singular value over the smallest one, might be extremely high if the initial conditions of the optimization process are improperly set. All of these might explain why, with the exception of two studies proposing optimal exciting static postures to identify the CoM parameters, no optimal dynamics motion have been proposed for humanoids [15], [16]. In these studies, the total condition number of the regressor of the CoM model has been used [15]. Baelemans et al. [15] generated a very large number of feasible exciting static postures for estimating the robots CoM position. In their approach, the relative position of the 


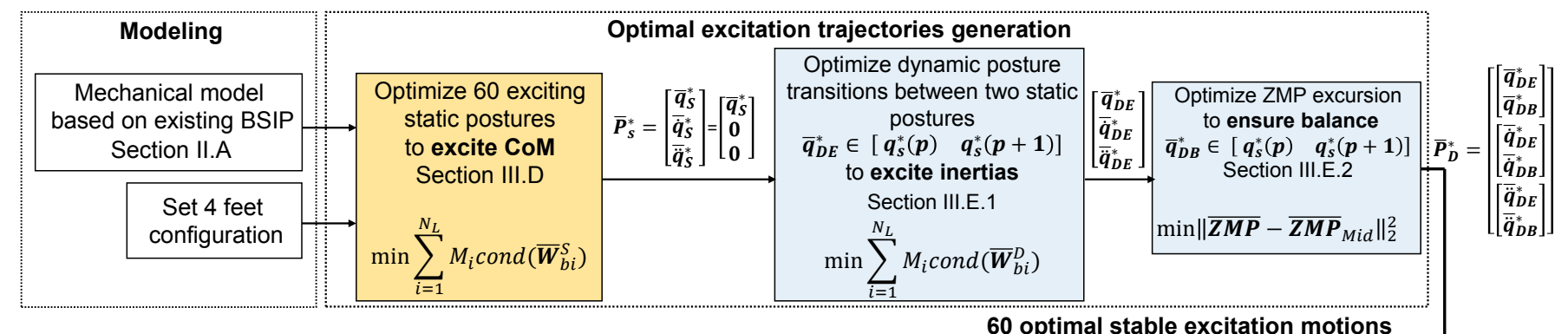

60 optimal stable excitation motions

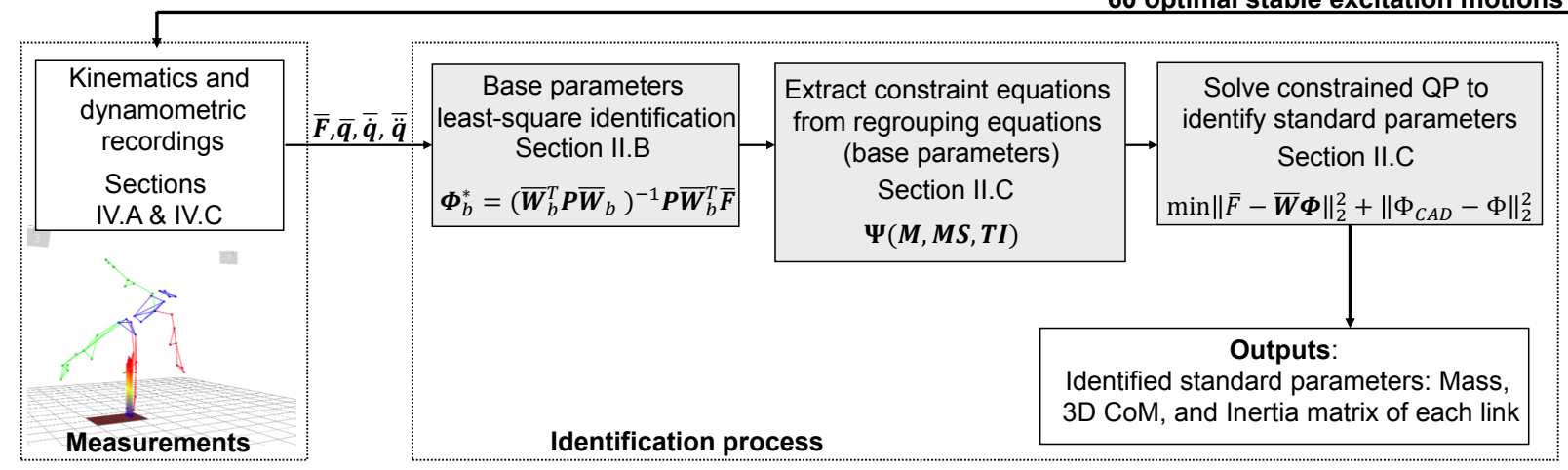

Fig. 1. Complete overview of the proposed method. Optimal static postures are generated (orange block) to excite the CoM parameters. In-between each static posture, a feasible exciting motion is generated (blue blocks) to excite inertias. Kinematic and dynamometric data collected during the optimal exciting motions are used in a constrained QP (grey blocks) to determine each link' mass, CoM and inertia matrix.

feet was not constrained. This meant that the robot had to be manually repositioned in a cumbersome and time consuming maneuver. Mayr et al. [16] proposed a very simple idea to get rid of the force sensor and balance constraint by supporting the robot using a mast at waist level. Using the fact that the sum of the external forces acting on the robot were balanced and by means of a constrained quadratic program (QP) they could generate motions for the identification of each segment's CoM. This idea is very interesting but cannot be practically applied to human subjects for which the static hypothesis would not be valid. For them, Bonnet and Venture have proposed a visual biofeedback system displaying optimal exciting trajectories designed to identify all inertial parameters in less than one minute while taking into account the subject's physical limitations [14]. This approach, also based on a constrained QP, has successfully been used to identify all the SIP of a simplified human planar model. In this context, new methods to automatically generate optimal exciting motions and to identify physically consistent SIP would be of a great help in numerous applications involving anthropomorphic systems.

\section{B. Paper contribution}

The method described in this study provides an all-inclusive framework to identify the standard parameters of legged systems. In the case of human subjects it provides normalized and repeatable motion information trough a visual biofeedback system. For humanoids this study provides a continuous optimal exciting trajectory to identify the standard inertial parameters without manual intervention. The resultant continuous trajectory can be seen as a set of optimal sequences forming an optimal choreography or, as we call it, as an optimal exciting dance. The main steps of the method are summarized in Fig. 1 and the paper is organized as follows. Section II.A presents the human and robot mechanical models. Section II.B (grey blocks in Fig. 1) describes the identification model including the first contribution of the paper 1) the 3D extended constrained QP to identify standard inertial parameters. Section III (orange and blue blocks in Fig. 1) details the two other contributions of the paper 2) a new method to generate 3D optimal and feasible exciting static and dynamic (sections III.D and III.E respectively) postures and 3) introduces a new optimal criterion to describe the excitation. Section IV presents the experimental results obtained with a human subject and the HOAP-3 humanoid robot. Finally, the paper ends by discussing the advantages and limitations of this method.

\section{IDENTIFICATION MODEL}

\section{A. Modelling}

1) Mechanical Model: To exemplify our method two anthropomorphic structures were considered. First a biomechanical model of a human (height $=1.75 \mathrm{~m}$; weight $=65 \mathrm{~kg}$ ) consisting of $N_{L}=12$ rigid segments, articulated by $N_{J}=23 \mathrm{DoF}$ (Fig. 2.b) was developed. The segment lengths and initial SIP were set using available AT [2]. Joint angle and torque limitations were adapted to the proposed model from literature values [17]. A second model representing the mechanical structure of a Fujitsu HOAP-3 humanoid robot (size: $0.88 \mathrm{~m}$; weight: $7.9 \mathrm{~kg}$; $N_{L}=12$ rigid segments; $N_{J}=21$ DoF ; Fujitsu-Siemens) was also developed (Fig. 2.a). 


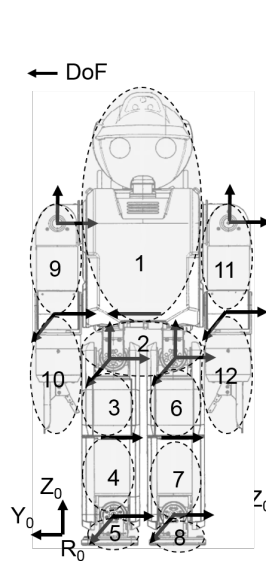

(a)

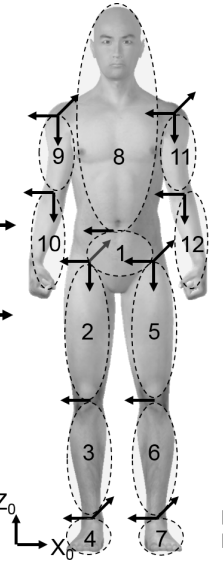

(b)

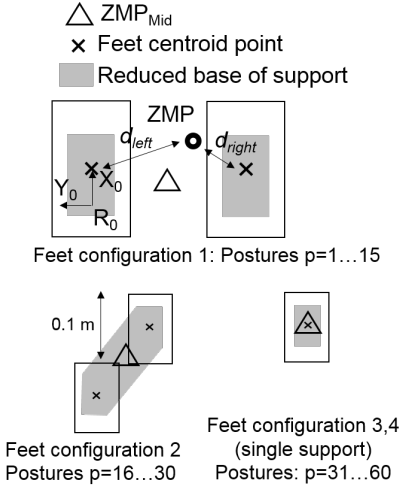

(c)
Fig. 2. The 12-bodied 3D models of (a) a HOAP-3 humanoid robot (21 DoF) and of (b) the human body $(23 \mathrm{DoF}))$. The four retained feet configurations and the principle for calculating force distribution under each foot (c).

The dynamic model of a floating base multi-body system, can be expressed as [20]:

$$
\left[\begin{array}{ll}
\mathbf{H}_{w w} & \mathbf{H}_{w c} \\
\mathbf{H}_{c w} & \mathbf{H}_{c c}
\end{array}\right]\left[\begin{array}{c}
\ddot{\mathbf{q}} w \\
\ddot{\mathbf{q}}
\end{array}\right]+\left[\begin{array}{l}
\mathbf{b}_{w} \\
\mathbf{b}_{c}
\end{array}\right]=\left[\begin{array}{l}
\mathbf{0} \\
\boldsymbol{\Gamma}
\end{array}\right]+\sum_{k=1}^{N_{c}}\left[\begin{array}{l}
\mathbf{J}_{w_{k}}^{T} \\
\mathbf{J}_{c_{k}}^{T}
\end{array}\right] \mathbf{F}_{k}
$$

where the upper part of the equation represents the root-link dynamics, and the lower part accounts for the other chains segment dynamics.

- $\mathbf{H}_{w w}(6 \times 6)$ and $\mathbf{H}_{w c}\left(6 \times N_{J}\right)$ are the root-link inertia matrices; $\mathbf{H}_{c w}\left(N_{J} \times 6\right), \mathbf{H}_{c c}\left(N_{J} \times N_{J}\right)$ are the chains segments inertia matrices;

- $\ddot{\mathbf{q}}_{w}$ denotes the $(6 \times 1)$ linear and angular acceleration vector of the root-link in the global system of reference;

- $\ddot{\mathbf{q}}$ and $\Gamma$ are the $\left(N_{J} \times 1\right)$ joint acceleration and torque vectors, respectively;

- $\mathbf{b}_{w}(6 \times 1)$ and $\mathbf{b}_{c}\left(N_{J} \times 1\right)$ are the bias force vectors describing centrifugal, Coriolis, and gravity forces of the root-link and of the chain segments, respectively;

- $N_{c}$ is the number of contact points with the environment;

- $\mathbf{J}_{w_{k}}$ and $\mathbf{J}_{c_{k}}$ are the Jacobian matrices expressed at contact point $k$ that map external wrenches $\mathbf{F}_{k}=$ $\left[F_{X_{k}} F_{Y_{k}} F_{Z_{k}} M_{X_{k}} M_{Y_{k}} M_{Z_{k}}\right]^{T}$ to the root-link and chains segments, respectively.

2) External wrench distribution and dynamic balance: The Zero-Moment-Point (ZMP) is the point on the ground at which the resultant tangential moments of the active forces are null [21]. The robot is stable when the ZMP is maintained within the polygon of support. The ZMP can be calculated from the total external wrench $\mathbf{F}_{0}$ acting on the root-link and expressed in the global system of reference using the inverse kinematic model of the supporting leg [22]. In this study, it is assumed that the feet configuration, and thus the convex hull of the base of support and its centroid point $Z M P_{M i d}$, are known for a given motion. Four feet configurations were imposed as described in Fig. 2.c.
In order to estimate joint torques it is necessary to know the wrench applied at each contact point. A rough estimate of the force distribution under each foot during double support can be obtained by using a linear relationship between the position of the total ZMP and the centroid point of each foot. This method was first introduced by Xiang et al. [22] and allows to estimate the external wrench under right, $\mathbf{F}_{\text {right }}$, and left, $\mathbf{F}_{\text {left }}$, feet as a function of $\mathbf{F}_{0}$ :

$$
\left(\begin{array}{c}
\mathbf{F}_{\text {right }} \\
\mathbf{F}_{\text {left }}
\end{array}\right)=\mathbf{F}_{0}\left(\begin{array}{c}
\frac{d_{\text {right }}}{d_{\text {right }}+d_{\text {left }}} \\
\frac{d_{\text {left }}}{d_{\text {right }}+d_{\text {left }}}
\end{array}\right)
$$

where $d_{\text {left }}$ and $d_{\text {right }}$ are the distances between the total ZMP and the centroid point under left and right feet (Fig. 2.c), respectively.

\section{B. Inertial parameters identification}

Two steps are required for the identification procedure. First, the base parameters need to be estimated. Secondly, the regrouping equations of the base parameters are used in a constrained quadratic programming (QP) to identify $\boldsymbol{\Phi}$ the vector of SIP, containing all the individual links masses, CoMs and inertias.

1) Linear identification model: The equations of motion are linear with respect to the dynamics parameters expressed in the joint frame [23]. Because of this property the $\left(\left(N_{J}+6\right) \times\right.$ $10 N_{L}$ ) observation matrix, also called regressor, can be built and (1) then rewritten as:

$$
\left[\begin{array}{l}
\mathbf{W} \\
\mathbf{C}
\end{array}\right] \boldsymbol{\Phi}=\left[\begin{array}{l}
\mathbf{0} \\
\boldsymbol{\Gamma}
\end{array}\right]+\sum_{k=1}^{N_{c}}\left[\begin{array}{l}
\mathbf{J}_{w_{k}}^{T} \\
\mathbf{J}_{c_{k}}^{T}
\end{array}\right] \mathbf{F}_{k}
$$

where $\mathbf{W}\left(6 \times 10 N_{L}\right)$ and $\mathbf{C}\left(N_{J} \times 10 N_{L}\right)$ are the regressors of the root-link and of the chains respectively; $\Phi\left(10 N_{L} \times 1\right)$ is the vector of standard inertial parameters to identify, $\boldsymbol{\Phi}=\left[\boldsymbol{\Phi}_{1}^{T} \ldots \boldsymbol{\Phi}_{N_{L}}^{T}\right]^{T}$. For each segment $i$, 10 inertial parameters can be expressed in the joint frame $\boldsymbol{\Phi}_{i}=\left[\begin{array}{lll}M_{i} & \mathbf{M S}_{i} & \mathbf{T I}_{i}\end{array}\right]$, where $M_{i}$ is the mass, $\mathbf{M S}_{i}=$ $\left[\begin{array}{lll}M S X_{i} & M S Y_{i} & M S Z_{i}\end{array}\right]$ is the 3-dimension vector of the first moment of inertia, and the 6-dimension vector $\mathbf{T I}_{i}=$ $\left[\begin{array}{llllll}X X_{i} & Y Y_{i} & Z Z_{i} & X Y_{i} & X Z_{i} & Y Z_{i}\end{array}\right]$ gathers the components of the $3 \times 3$ tensor of inertia.

2) Base parameters identification: The upper part of (3) has been shown [3] to be independent of $\Gamma$ and can be used to identify $\boldsymbol{\Phi}$, the vector containing the standard inertial parameters (SIP). However, since $\mathbf{W}$ is not a full column rank matrix, a direct least squares approach is not suitable for its solution. We find in the robotics system identification the literature that this equation can be rewritten using the $s o$ called base parameters (BP) as defined in [18] in such a way as to have a full column rank regressor. In this way, vector $\boldsymbol{\Phi}_{b}$ $\left(N_{B} \times 1\right)$ is the minimal identifiable set of inertial parameters required to describe the dynamics of the system. Since the BP are intrinsically related to the kinematic structure of the system they can be computed numerically [24]. Their computation consists in finding the equivalent regressor $\mathbf{W}_{b}\left(6 \times N_{B}\right)$ that is a full column rank matrix by combining the linearly dependent 
columns. This results in the elimination and regrouping of the SIP into the vector $\boldsymbol{\Phi}_{b}$, and the upper part of (3) can be written as (see [18], [23]):

$$
\mathbf{W} \boldsymbol{\Phi}=\mathbf{W}_{b} \boldsymbol{\Phi}_{b}=\sum_{k=1}^{N_{c}} \mathbf{J}_{w_{k}}^{T} \mathbf{F}_{k}
$$

The numerical approach is well-suited when different BP sets, i.e. static or dynamic ones, need to be selected as described in section III. For example, when in a static situation, the inertias are automatically removed by setting the joints and root-link velocities and accelerations to zero; the obtained BP are then only a function of the masses and of the CoM. That is, the columns of the regressor corresponding to the inertias are automatically removed from the calculation. The remaining static parameters are then regrouped, depending on the kinematics of the system, as follows:

$$
\begin{aligned}
\boldsymbol{\Phi}_{b i}= & {\left[M_{b i} \mathbf{M S}_{b i}^{T} \mathbf{T} \mathbf{I}_{b i}^{T}\right]^{T} \text { root link } } \\
\boldsymbol{\Phi}_{b i}= & {\left[M S X_{b i} M S Y_{b i} X X_{b i}-Y Y_{b i} Z Z_{b i}\right.} \\
& \left.Y Z_{b i} Z X_{b i} X Y_{b i}\right]^{T} \text { hinge joint } \\
\boldsymbol{\Phi}_{b i}= & {\left[\mathbf{M S}_{b i}^{T} \mathbf{T} \mathbf{I}_{b i}^{T}\right]^{T} \text { spherical joint } }
\end{aligned}
$$

where $M_{b i}$ is the BP of link $i$ representing the sum of the masses of links that are children of link $i$ in the chain: $M_{b i-1}=M_{i-1}+M_{b i} ; \mathbf{M S}_{b i}=\left[\begin{array}{lll}M S X_{b i} & M S Y_{b i} & M S Z_{b i}\end{array}\right]$ the BP of link $i$ representing the sum of the first moment of inertia, and depending only of inertial parameters of links that are children of link $i$ in the chain; $\mathbf{T I}_{b i}=$ $\left[\begin{array}{llllll}X X_{b i} & Y Y_{b i} & Z Z_{b i} & Y Z_{b i} & Z X_{b i} & X Y_{b i}\end{array}\right]$ the BP of link $i$ representing the inertia, and depending only on the inertial parameters of link $i$ and its children in the chain. Sub-regressor matrices can be created from the base parameters depending on their type: static (Mass, CoM) or dynamic (inertias). In this study we propose to create both the static $\mathbf{W}_{b i}^{S}$ and dynamic $\mathbf{W}_{b i}^{D}$ BP regressor matrices for each link $i$. They are created by selecting the columns of $\mathbf{W}_{b}$ that correspond to $\mathbf{M}_{b i}$ and $\mathbf{M S}_{b i}$ parameters, and to $\mathbf{T I}_{b i}$, respectively.

Sampling (4) over a given motion, the identification problem for $n$ time-samples becomes:

$$
\begin{gathered}
{\left[\begin{array}{c}
\mathbf{W}_{b}(1) \\
\vdots \\
\mathbf{W}_{b}(n)
\end{array}\right] \boldsymbol{\Phi}_{b}=\sum_{k=1}^{N_{c}}\left[\begin{array}{c}
\mathbf{J}_{w_{k}}^{T}(1) \mathbf{F}_{k}(1) \\
\vdots \\
\mathbf{J}_{w_{k}}^{T}(n) \mathbf{F}_{k}(n)
\end{array}\right]} \\
\overline{\mathbf{W}}_{b} \boldsymbol{\Phi}_{b}=\overline{\mathbf{F}}
\end{gathered}
$$

and can be solved using a weighted Moore-Penrose pseudoinverse matrix:

$$
\boldsymbol{\Phi}_{b}^{*}=\left(\overline{\mathbf{W}}_{b}^{T} \mathbf{P} \overline{\mathbf{W}}_{b}\right)^{-1} \mathbf{P} \overline{\mathbf{W}}_{b}^{T} \overline{\mathbf{F}}
$$

where $\mathbf{P}$ is a weight matrix. Indeed, the elements of $\overline{\mathbf{F}}$ are expressed in different units and of different orders of magnitude and it is preferable to use a weighted least-square method based on the calculation of the relative standard deviation of the identified parameters [25]. The relative standard deviation $\sigma_{\%}$ of the identified parameters gives an image of the accuracy of the estimated BP values and it is calculated according to [25].

\section{Standards inertial parameters identification}

While the full set of SIP $\boldsymbol{\Phi}$ is necessary to compute the forward dynamics and joint torques (lower part of (1) and (3)), some of them fall in the null-space of the regressor; there is no direct way to identify them. Several methods have been proposed in the literature and each use a priori knowledge assuming physical consistency [26], [25]. The present study proposes to extend the method proposed by Bonnet and Venture [14] for its use in the 3D case. Here, a hybrid cost function and a constrained QP allow the least-square fitting of recorded external wrench while minimizing the deviation between the estimated SIP and their CAD or AT values. We formulate the QP as:

$$
\text { Find } \boldsymbol{\Phi}^{*} \in \min _{\boldsymbol{\Phi}}\|\overline{\mathbf{F}}-\overline{\mathbf{W}} \boldsymbol{\Phi}\|_{2}^{2}+\left\|\boldsymbol{\Phi}_{C A D}-\boldsymbol{\Phi}\right\|_{2}^{2}
$$

subject to

$$
\begin{aligned}
& M_{i} \geq 0 \\
& C o M_{i j}^{-} \leq C o M_{i j} \leq C o M_{i j}^{+} \quad j=x, y, z \\
& \mathbf{v}^{T} I_{i} \mathbf{v}>\epsilon \\
& \text { with } \quad i=1, \ldots, N_{L} \\
& \mathbf{\Phi}_{b}^{*}=\Psi(\mathbf{M}, \mathbf{M S}, \mathbf{T I})
\end{aligned}
$$

where $\boldsymbol{\Phi}_{C A D}\left(10 N_{L} \times 1\right)$ refers to the SIP values obtained from CAD or AT, and $\boldsymbol{\Phi}^{*}\left(10 N_{L} \times 1\right)$ to the estimated ones.

In order to ensure physical consistency a constraint stating that the all masses must be positive was included ( $i: M_{i} \geq 0$ ). Additionally, the CoM position of each link was constrained to be inside of the link's specific oriented bounding box (defined in the local link frame):

$$
C o M_{i j}^{-} \leq C o M_{i j} \leq C o M_{i j}^{+} \quad j=x, y, z
$$

where $\mathrm{CoM}_{i j}^{-}$and $\mathrm{CoM}_{i j}^{+}$are the upper and lower bounds of the oriented bounding box attached to the $i^{\text {th }}$ link.

The inertia matrices $I_{i}$ were constrained to be positive definite, i.e. for every non-zero vector $\mathbf{v} \in \mathbb{R}^{3}, \mathbf{v}^{T} I_{i} \mathbf{v}>0$. This formulation is semi-infinite and was approximated by using a small positive tolerance value $\epsilon$ such as $\mathbf{v}^{T} I_{i} \mathbf{v}>\epsilon$, and $\epsilon$ set to $10^{-3}$. For each vector $\mathbf{v}_{j}$, uniformly distributed over the unit sphere, we get a linear inequality in $\boldsymbol{\Phi}$ defined by:

$$
\left[\begin{array}{c}
v_{x j}^{2} \\
2 v_{x j} v_{y j} \\
2 v_{x j} v_{z j} \\
v_{y j}^{2} \\
2 v_{y j} v_{z j} \\
v_{z j}^{2}
\end{array}\right]^{T}\left[\begin{array}{c}
X X \\
X Y \\
X Z \\
Y Y \\
Y Z \\
Z Z
\end{array}\right] \geq \epsilon
$$

The last set of constraints is composed of the regrouping relations (5) between the identified BP and SIP and expressed as a set of linear constraints [14]: 
TABLE I

CORRESPONDENCE BETWEEN THE POSTURE NUMBER $p$, THE TYPE OF SUPPORT AND THE ROLE OF EACH LIMB: EXCITATION OR BALANCE

\begin{tabular}{|c|c|c|c|c|}
\hline Posture $p$ & $\in\left[\begin{array}{ll}1 & 15\end{array}\right]$ & $\in\left[\begin{array}{lll}16 & 30\end{array}\right]$ & $\in\left[\begin{array}{ll}31 & 45\end{array}\right]$ & $\in\left[\begin{array}{ll}46 & 60\end{array}\right]$ \\
\hline Support & Double & $\begin{array}{l}\text { Double } \\
\text { right forward }\end{array}$ & $\begin{array}{l}\text { Single } \\
\text { right }\end{array}$ & $\begin{array}{l}\text { Single } \\
\text { left }\end{array}$ \\
\hline $\begin{array}{l}\text { Joints used for } \\
\text { excitation } \overline{\mathbf{q}}_{D E}\end{array}$ & $\begin{array}{l}\overline{\mathbf{q}}_{\text {Dtrunk }} \\
\overline{\mathbf{q}}_{\text {Darms }}\end{array}$ & $\begin{array}{l}\overline{\mathbf{q}}_{\text {Dtrunk }} \\
\overline{\mathbf{q}}_{\text {Darms }}\end{array}$ & $\overline{\mathbf{q}}_{\text {Dlleg }}$ & $\overline{\mathbf{q}}_{\text {Drleg }}$ \\
\hline $\begin{array}{l}\text { Joints used for } \\
\text { balance } \overline{\mathbf{q}} \overline{\mathbf{A}}_{D B}\end{array}$ & $\begin{array}{l}\overline{\mathbf{q}} \text { Dlleg } \\
\overline{\mathbf{q}}_{\text {Drleg }}\end{array}$ & $\begin{array}{l}\overline{\mathbf{q}}_{\text {Dlleg }} \\
\overline{\mathbf{q}}_{\text {Drleg }}\end{array}$ & $\begin{array}{l}\overline{\mathbf{q}} \text { Dtrunk } \\
\overline{\mathbf{q}}_{\text {Darms }} \\
\overline{\mathbf{q}}_{\text {Drleq }}\end{array}$ & $\begin{array}{l}\overline{\mathbf{q}} \text { Dtrunk } \\
\overline{\mathbf{q}}_{\text {Darms }} \\
\overline{\mathbf{q}}_{\text {Dlleq }}\end{array}$ \\
\hline $\begin{array}{l}\text { Nbr of link } N_{L D E} \\
\text { Nbr of joint } N_{J D E} \\
\text { Nbr of joint } N_{J D B}\end{array}$ & $\begin{array}{c}6 \\
9 \\
12\end{array}$ & $\begin{array}{c}6 \\
9 \\
12\end{array}$ & $\begin{array}{c}3 \\
6 \\
15\end{array}$ & $\begin{array}{c}3 \\
6 \\
15\end{array}$ \\
\hline
\end{tabular}

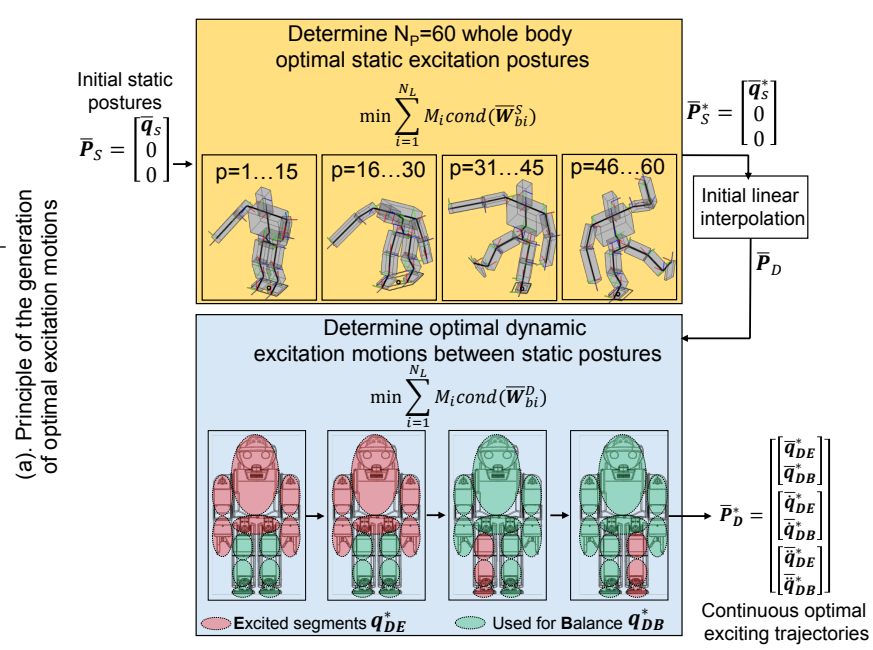

$$
\mathbf{\Phi}_{b}^{*}=\Psi(\mathbf{M}, \mathbf{M S}, \mathbf{T I})
$$

where $\boldsymbol{\Phi}_{b}^{*}$ is the identified vector of base parameters and $\Psi$ are the corresponding symbolic equations function of SIP. An example of symbolic equation is given in section III.C. Any BP that could not be identified with a standard deviation inferior to $10 \%$ was discarded from the QP.

\section{OPTIMAL EXCITING MOTIONS}

Moving an anthropomorphic structure requires solving numerous constraints as it is usually composed of more than twenty DoFs with limited actuation capabilities, is intrinsically unstable, and is prone to auto-collisions. Also, for any identification process, hundreds of samples should be considered per trajectory. The automatic generation of optimal exciting motions for such a system is not trivial. The large problem size and the difficulty in choosing initial conditions that satisfy all the constraints often lead to unfeasible solutions or to a local minima. This convergence issue was addressed by decoupling the determination of optimal postures by exciting the static parameters (masses, CoMs), and the dynamic parameters (inertias), separately. This was achieved with the use of a new criterion built from the base parameters sub-regressor matrices. The orange and blue blocks in Fig. 1 present an overview of the proposed method for optimal exciting motions generation. Fig. 3.a gives a more detailed representation of this method. For each of the four feet configurations represented in Fig. 2.c and described in Table I, we generate a number $N_{p}=1 \ldots p$ of static optimal postures, $\overline{\mathbf{P}}_{S}^{*}=\left[\begin{array}{lll}\overline{\mathbf{q}}_{S}^{*} & 0 & 0\end{array}\right]^{T}$, where $\overline{\mathbf{q}}_{S}^{*}$ is of size $\left(N_{J} \times N_{p}\right)$, that aim at exciting the CoMs (orange block in Fig. 3.a).

These static postures are joined by optimal motions $\overline{\mathbf{P}}_{D}^{*}$. As presented in the blue block of the lower part of Fig. 3.a, all links move simultaneously, some are used for maintaining dynamical balance while others optimally excite their inertias. At the bottom of Fig. 3.a, the joints, $\overline{\mathbf{q}}_{D B}^{*}$, of the green links are used to maintain balance while the joints of the red links, $\overline{\mathbf{q}}_{D E}^{*}$, are moving in an optimal exciting way. $\overline{\mathbf{q}}_{D B}^{*}$ and $\overline{\mathbf{q}}_{D E}^{*}$ are both subsets of $\overline{\mathbf{q}}_{D}^{*}=\left[\begin{array}{ll}\overline{\mathbf{q}}_{D E}^{*}{ }^{T} & \overline{\mathbf{q}}_{D B}^{*}{ }^{T}\end{array}\right]^{T}$. The feet configuration determines which links should be excited and

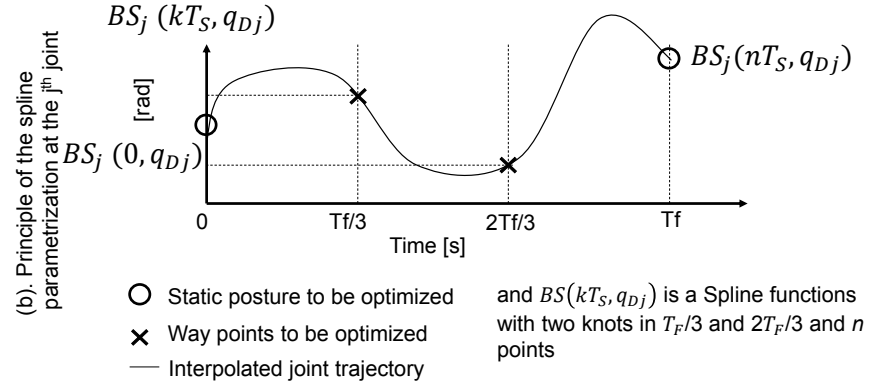

Fig. 3. Overview of the multi-level optimization process used to determine the optimal exciting motions while ensuring mechanical limitations and dynamic balance (a). B-spline parameterization used to represent joint trajectories (b)

which ones should be used for balance (Table I). In Table I, $q_{\text {Dtrunk }}, q_{\text {Darms }}, q_{\text {Drleg }}$, and $q_{\text {Dlleg }}$ refer to dynamic motion of the joints of the trunk, both arms, right leg, and left leg, respectively. $N_{L D E}, N_{J D E}$ are the number of links and joints to be excited, and $N_{J D B}$ is the number of joints used to maintain balance. For example, for the postures where the two feet are on the ground $(p \in[130])$, the trunk and the arms are moving optimally, i.e. exiting their inertias, while the two legs are used to maintain dynamic balance. Thus, for $p \in\left[\begin{array}{ll}1 & 30\end{array}\right], \overline{\mathbf{q}}_{D}^{*}=$ $\left[\begin{array}{lll}\overline{\mathbf{q}}_{D E}^{*}{ }^{T} & \overline{\mathbf{q}}_{D B}^{*}{ }^{T}\end{array}\right]^{T}=\left[\left[\begin{array}{lll}\overline{\mathbf{q}}_{\text {Dtrunk }}^{*} & \overline{\mathbf{q}}_{\text {Darms }}^{*}\end{array}\right]\left[\begin{array}{lll}\overline{\mathbf{q}}_{\text {Dlleg }}^{*} & \overline{\mathbf{q}}_{\text {Drleg }}^{*}\end{array}\right]\right]^{T}$. To ensure continuity between sets of postures with different feet configurations, an intermediate posture (between 15 and 16, 30 and 31, and 45 and 46) was inserted, forcing the model to come back to a double support. The dynamic balance can be decoupled from the identification of the SIP due to the fact that it is not directly linked to the position of the center of pressure [15].

\section{A. B-spline trajectory parameterization for dynamics posture}

For the dynamic case, it is preferable to interpolate joint trajectories [12] in order to calculate the excitation criterion (see III-B) and to accurately estimate joint velocity and acceleration. Thus, to reduce the size of the optimization problem, B-splines were used to interpolate the trajectory of each joint [12]. The posture transition time $T_{F}$ required to move from 
one posture $\mathbf{P}_{S}^{*}(p)$ to the next $\mathbf{P}_{S}^{*}(p+1)$ was arbitrarily set to comply with maximal joint velocity, and was set to $T_{F}=2 \mathrm{~s}$ and $T_{F}=5 \mathrm{~s}$ for the human subject and the humanoid robot respectively. The number of via points was set to $N_{K}=2$ for both models. During the optimization, between $\mathbf{P}_{S}^{*}(p)$ and $\mathbf{P}_{S}^{*}(p+1)$, joint angles $\left(B S_{j}\left(k T_{S}, q_{D_{j}}\right), k=1, . .,(n-1)\right)$, velocities $\left(B \dot{S}_{j}\left(k T_{S}, q_{D j}\right)\right)$, and accelerations $\left(B \ddot{S}_{j}\left(k T_{S}, q_{D j}\right)\right)$ were interpolated at $50 \mathrm{~Hz}\left(T_{S}=0.02 \mathrm{~s}\right.$ and $\left.n=T_{F} / T_{S}\right)$ by passing through the via points as shown in Fig. 3.b. Trajectories were also constrained to have a null initial and final velocity and acceleration. Finally, all joints motions were set to start and finish synchronously. Sixth order B-splines were used to ensure continuous acceleration and kinematic constraints.

\section{B. Excitation criterion}

As previously stated, several cost functions have been proposed to determine the optimal exciting motions for serial manipulators [12]. Since the sensitivity of a linear least square problem for estimating parameters can be measured using the regressor's condition number, it has been extensively used for this purpose. This was done here with the condition number of the regressor linking the inertial parameters to the measured joint torques (the matrix $\mathbf{C}$ in the lower part of (3)). However, this metric should only be used when the regressor is relatively well equilibrated [13]. Presse and Gautier [13] proposed an intuitive weighting method, using prior knowledge on the SIP obtained from CAD data, to scale and normalize all parameters. Otherwise, small link parameters being more difficult to identify, as they have a smaller influence on the measured dynamics quantities, will lead to a ill-conditioned regressor [13]. This was demonstrated with serial industrial robots [13] that commonly use joint torques as a dynamic measured quantity. Joint torque, provides at least one measurement at each link level and is, by definition, a richer signal than the resultant vector of the external wrench. Consequently, even a small parameter will produce a readable, although noisy, change in the signal. For the human case joint torques cannot be measured and most humanoid robots do not have joint torque sensors, this led us to work with the external wrench expressed at the root-link instead of using joint torques. Additionally, in a case of an anthropomorphic structure the weight ratio between the smaller links (feet, hands) and the larger one (trunk) is very important, as is their influence on the resultant external wrench. Also, initial conditions of the optimization problem must be chosen wisely in order to avoid local minimum and numerical instabilities. These conditions are not trivially set for an anthropomorphic structure since it also must be dynamically stable, avoid autocollision, and respect several mechanical constraints (the maximal joint torques of a humanoid robot are much lower than the ones of a serial manipulator). Consequently, the problem of an ill conditioned regressor is emphasized for such systems. To overcome this problem we propose to adapt a numerical method initially proposed to select exciting motions from an existing human motion database [8]. The idea is to divide the total $\mathrm{BP}$ regressor $\mathbf{W}_{b}$ into sub-regressors containing only information corresponding to certain links or groups of links (head, arms, right leg) or type of parameters (CoM, inertias). This can be done by choosing the corresponding columns of $\mathbf{W}_{b}$ (see section II.B for regressor and base parameters definition). Using the BP regrouping relations given by (5), it is possible to calculate the sub-regressor $\overline{\mathbf{W}}_{b i}$ for each link $i$. The condition number of each sub-regressor can then be computed to evaluate the excitation of each motion separately. In this way, even a relatively poorly excited link will give a relatively small condition number and avoid numerical issues. Additionally, similarly to Gautier et al. [13], we propose to multiply each condition number by the mass of the corresponding segment which gives more importance to larger links. With this criterion it is also very easy to generate exciting motions for individual links or parameters (static, i.e. CoM, or dynamic, i.e. inertia). In this context, we propose a new criterion that is the mass weighted sum of the condition numbers of the sub-regressors:

$$
J_{e x c}=\sum_{i=1}^{N_{L}} M_{i} \operatorname{cond}\left(\overline{\mathbf{W}}_{b i}\right)
$$

where cond refers to the condition number calculated using a SVD decomposition.

\section{Numerical analysis of the excitability criterion}

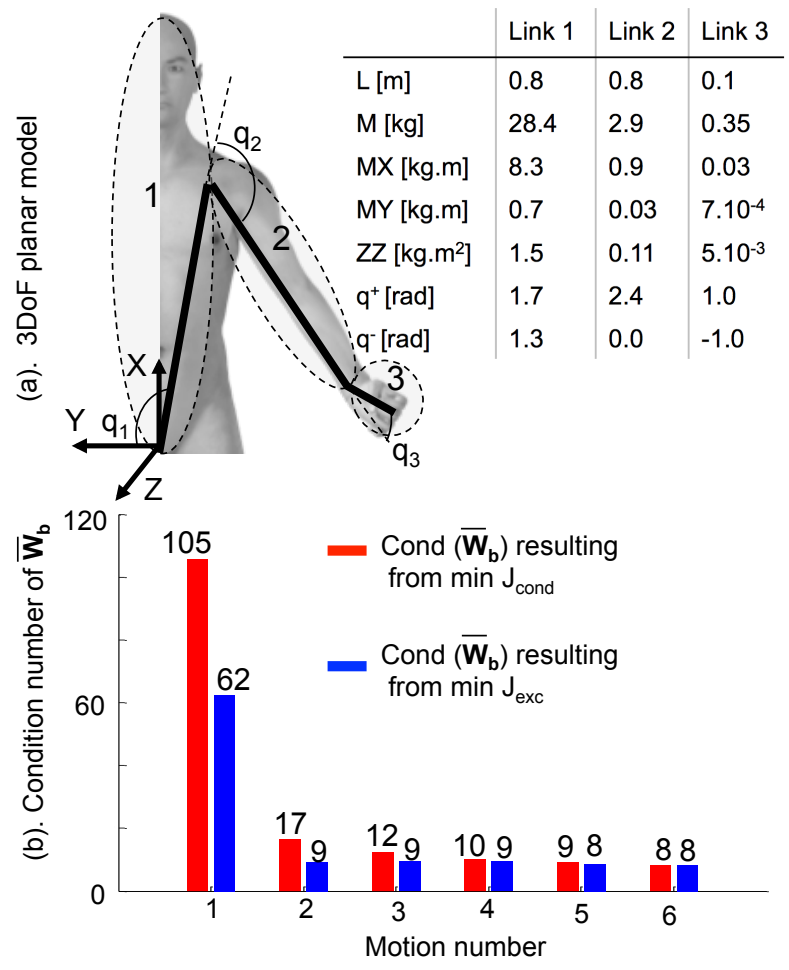

Fig. 4. 3 DoF planar mechanical model used for the numerical analysis of the excitation criterion (12)(a). Simulation results showing that the proposed criterion converges faster and provides a better excitation than when minimizing the condition number of the total BP regressor (b). 
The purpose of this sub-section is to highlight the benefit of the proposed excitability criterion (12) for a simplified $N_{J}=3$ DoF planar model, described in Fig. 4.a. This model mimics a system composed of the head-trunk, arm, and hand in the frontal plane and its corresponding anthropomorphic measurements [2] as indicated in Fig. 4.a. Each link was provided with a mass, 2-D COM position and one inertia for a total of 12 SIP. The base parameters $\boldsymbol{\Phi}_{\mathbf{b}}$ of this simple model were calculated using the previously mentioned numerical method [24]. The 10 BP of this model are the following:

$$
\begin{aligned}
& \operatorname{link}_{1}\left\{\begin{array}{l}
\Phi_{b}(1)=M_{1 R}=M_{1}+M_{2}+M_{3} \\
\Phi_{b}(2)=M X_{1 R}=M_{X 1}+0.8 M 2+0.8 M_{3} \\
\Phi_{b}(3)=M Y_{1} \\
\Phi_{b}(4)=Z Z_{1 R}=Z Z_{1}+0.64 M_{2}+0.64 M_{3}
\end{array}\right. \\
& \operatorname{link}_{2}\left\{\begin{array}{l}
\Phi_{b}(5)=M X_{2 R}=M X_{2}+0.2 M_{3} \\
\Phi_{b}(6)=M Y_{2} \\
\Phi_{b}(7)=Z Z_{2 R}=Z Z_{2}+0.04 M_{3}
\end{array}\right. \\
& \operatorname{link}_{3} \begin{cases}\Phi_{b}(8) & =M X_{3} \\
\Phi_{b}(9) & =M Y_{3} \\
\Phi_{b}(10) & =Z Z_{3}\end{cases}
\end{aligned}
$$

where the index $R$ stands for regrouped. The first four BP are related to the first link. BP 5, 6, and 7 are related to the second link, and the last three terms of $\boldsymbol{\Phi}_{\mathbf{b}}$ correspond to the SIP of the last link, excluding the mass, that can be identified separately. From these observations, three subregressor matrices, one for each link, can be built by using columns of $\mathbf{W}_{b}$.

Definition 1: Let us define $\mathbf{W}_{b}^{c}$ as a matrix composed of $l$ column vectors of $\mathbf{W}_{b}$ matrix corresponding to the column numbers defined by the $l$-tuple $c=\left\{n c_{1}, n c_{2}, . ., n c_{l}\right\}$.

Based on the Definition 1, we extract $\mathbf{W}_{b_{1}}^{c_{1}}, \mathbf{W}_{b_{2}}^{c_{2}}$ and $\mathbf{W}_{b_{3}}^{c_{3}}$ from $\mathbf{W}_{b}$ respectively with $c_{1}=\{1,2,3,4\}, c_{2}=\{5,6,7\}$ and $c_{3}=\{8,9,10\}$. Note that the static (mass and CoM), $\mathbf{W}_{b}^{S}$, and dynamic (inertias), $\mathbf{W}_{b}^{D}$, sub-regressor matrices are also extracted from $\mathbf{W}_{b}$ by using Definition 1 such as $\mathbf{W}_{b}^{S}=\mathbf{W}_{b}^{c_{S}}$ and $\mathbf{W}_{b}^{D}=\mathbf{W}_{b}^{c_{D}}$ with $c_{S}=\{1,2,3,5,6,8,9\}$ and $c_{D}=\{4,7,10\}$. The benefit of the proposed cost function, $J_{\text {exc }}$, was highlighted by comparing the produced exciting motions with those obtained from the minimization of the condition number of the total BP regressor $J_{\text {cond }}=\operatorname{cond}\left(\overline{\mathbf{W}}_{b}\right)$ which is commonly used in industrial robotics [11]. The two optimization processes were to find the joint angle values at $N_{K}=4$ via points, equally distributed over the whole trajectory of $T_{F}=2 s$, that minimize the above mentioned criteria under the constraint that the B-spline interpolated joint trajectories respect the joint limitations $q_{j}^{-} \leq B S_{j}\left(k T s, q_{j}\right) \leq$ $q_{j}^{+}, \quad k=1, \ldots,(n-1)$. B-splines were used to interpolate the joint trajectories at $50 \mathrm{~Hz}$. The optimization problem was to determine $N_{J} N_{K}=12$ variables. The optimizations were run iteratively six times $($ iter $=1, \ldots, 6)$ by period of 2 s for a total of $12 \mathrm{~s}$. The number of rows of the regressor was augmented at each iteration with the regressors built from the previous optimizations. In this simulation, the external wrench was composed of 2 forces $\left(F_{X}, F_{Y}\right)$ and one resultant moment $\left(M_{Z}\right)$. Consequently, at each iteration iter the BP regressor $\overline{\mathbf{W}}_{b}$ was of size $((3 \times$ iter $\times(2 \times 50)) \times 10)$. After each optimization, and iteration, the condition number of the total BP regressor $\overline{\mathbf{W}}_{b}$ was calculated. As previously mentioned, the initial conditions of this problem are difficult to be set for anthropomorphic systems. A first approach is to have them vary linearly for each $j^{\text {th }}$ joint from their lower $\left(q_{j}^{-}\right)$to upper $\left(q_{j}^{+}\right)$limits. This produces joint trajectories that are not very exciting since joint accelerations are constants. This results in an initial BP regressor that is ill-conditioned with a condition number of $5.10^{16}$. Once optimized, the total condition number for the first iteration (from 0 to $T_{F}=2 s$ ) drops to $J_{\text {cond }}=105$ as represented in red in Fig. 4.b. Interestingly, when the proposed criteria $J_{e x c}$ is minimized, the condition number of the total BP regressor is even smaller and becomes $J_{\text {cond }}=62$. From this, it is clear that the proposed criterion produced a more exciting trajectory than the minimization of the condition number of the total BP regressor. Indeed, the ill-conditioned BP regressor leads to convergence problems in the optimization algorithm that were observed systematically when starting with poorly exciting initial conditions. However, these differences tend to decrease when the size of $\overline{\mathbf{W}}_{b}$ increases. One can see in Fig. $4 \mathrm{~b}$ that the proposed criteria converges to a global minimum faster than the minimization of the total BP regressor; however, they will both reach a minimum eventually. From this numerical analysis it can be concluded that the proposed criterion is a good candidate for reducing the problem of an ill-conditioned total $\mathrm{BP}$ regressor as it tends to a similar minimum while avoiding numerical instability and with a faster convergence.

\section{Static postures}

The floating base identification process uses three forces and three moments expressed at the root-link level. This means that six measurements are available to identify three CoM coordinates for each of the twelve links. As a rule of thumb, ten measurements should be recorded per parameter [13]. Thus, $N_{p}=60$ static postures $\overline{\mathbf{P}}_{S}^{*}$ are to be generated. An optimization process aiming to automatically determine these 60 optimal joint configurations $\overline{\mathbf{q}}_{S}^{*}\left(N_{J} \times 60\right)$ was developed for this purpose.

$$
\begin{aligned}
\text { Find } \overline{\mathbf{q}}_{s}^{*} \in \quad & \min _{\overline{\mathbf{q}}_{S} \in \mathbb{R}^{N_{J} \times 60}} \sum_{i=1}^{N_{L}} M_{i} \operatorname{cond}\left(\overline{\mathbf{W}}_{b i}^{S}\right) \\
\text { s.t. } \quad & q_{j}^{-} \leq \bar{q}_{S j} \leq q_{j}^{+} \\
& \overline{\dot{q}}_{S j}=\overline{\bar{q}}_{S j}=0 \\
& \left|\bar{\Gamma}_{j}\right| \leq \Gamma_{j}^{+} \\
& \text {with } \quad j=1, . ., N_{J} \\
& Z M P_{x, y}^{-} \leq Z \bar{M} P_{x, y} \leq Z M P_{x, y}^{+} \\
& 0 \leq \overline{\mathbf{d}}_{v e r t e x} \\
& \overline{\mathbf{P}}_{R F 1: 3_{x, y}}=\overline{\mathbf{P}}_{L F 1: 3_{x, y}}+\Delta \overline{\mathbf{P}}_{F}
\end{aligned}
$$

where $\overline{\mathbf{W}}_{b i}^{S}$ is built from the columns of the BP regressor corresponding to the CoM parameters only (cf. definition 1). 
During the optimization process all static postures must satisfy the the joint angles and gravity induced torque limits allowed for either the human subject [17] or the robot:

$$
\begin{gathered}
q_{S j}^{-} \leq \bar{q}_{S j} \leq q_{S j}^{+} \\
\left|\bar{\Gamma}_{j}\right| \leq \Gamma_{j}^{+}
\end{gathered}
$$

where $q_{j}^{-}, q_{j}^{+}, \Gamma_{j}^{+}$are lower and upper joint angle and maximal torque limitations, respectively.

Additionally, the anthropomorphic structure's balance must be guaranteed. This means that the ZMP should lie within the support polygon defined by the convex hull of the feet:

$$
Z M P_{x, y}^{-} \leq Z \bar{M} P_{x, y} \leq Z M P_{x, y}^{+}
$$

where $Z M P_{x, y}^{-}$and $Z M P_{x, y}^{+}$are the lower and upper boundaries of the base of support that are dependent on the feet configuration as represented in Fig. 2.c. Since we first consider the static case, the $Z M P_{x, y}$ is taken to be equivalent to the projection of the total CoM. Similarly to Baleaman et al. [15], the size of the base of support is reduced by $40 \%$ in all directions so as to account for inaccuracies in the robot control and other errors in the geometric parameters. Auto-collisions are avoided by defining a convex rectangular bounding box, represented on the upper part of Fig. 3.a, around each link and imposing the Euclidean distance, $d_{\text {vertex }}$, between the vertices of two boxes to always be positive $d_{\text {vertex }}>0$. The following collisions were checked: lower feet to the opposite upper and lower leg, lower arms to the trunk and upper leg and to the opposite lower arm. The feet relative position and orientation was kept constant for the postures that require double support (postures $p=1 \ldots 30$ ). This is achieved by setting the relative position of three points on each foot:

$$
\overline{\mathbf{P}}_{R F 1: 3_{x, y}}=\overline{\mathbf{P}}_{L F 1: 3_{x, y}}+\Delta \overline{\mathbf{P}}_{F}
$$

This constraint becomes inactive for single support postures (postures $p=31 \ldots 60$ ). $\overline{\mathbf{P}}_{R F}, \overline{\mathbf{P}}_{L F}$ are the absolute 3D position of the right and left foot respectively and are of size $3 \times 30 . \Delta \mathbf{P}_{F}(3 \times 30)$ sets the relative feet position depending on the posture. It is used to place the right foot in front of the left one during the static postures $p=16 \ldots 30 ; 0.1 \mathrm{~m}$ in case of the HOAP-3 or $0.2 \mathrm{~m}$ in case of the human subject. The sixty static postures thus generated were divided into four groups of fifteen postures. Each group having a different relative feet configuration, as represented on Fig. 2.c.

The initial feasible static postures $\overline{\mathbf{q}}_{S}$ were calculated using a custom inverse kinematic process to constrain the relative position of the feet and ensure static balance. The initial motions were reminiscent of a low amplitude squat while keeping a straight trunk. Initial postures of the arms were based on sinusoidal motions covering the whole joint space. A multi-start search [27] was run 20 times around the previously described initial static postures in order to avoid local minima. The average time of calculation to solve this problem was 270s.

\section{E. Dynamic postures transition}

Each motion between two successive static postures was calculated separately so as to reduce the dimension of the optimization problem. This means that the set of dynamic postures $\overline{\mathbf{P}}_{D}^{*}$ was computed for every two consecutive static postures $p$ and $p+1$. However, the results of the previous motions were included in the optimization process by augmenting (increasing the number of rows) the dynamic BP regressor. At posture $p$,

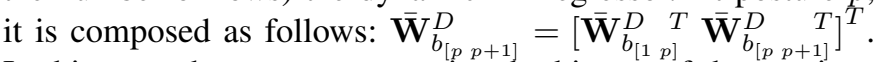
In this way, the regressor contains the history of the previous motions. The computation of the exciting trajectories of the upper and lower links were performed separately depending on each posture's foot placement (see section III and in Table I). In this way, the optimization problem was decomposed into two easier to solve sub-problems of lower dimension. The blue blocks in Fig. 1 show these processes. The first one, defined by (18), aims to determine the optimal exciting motions $\overline{\mathbf{q}}_{D E}\left(N_{J D E} \times N_{K}\right)$ by finding the $N_{K}=2$ optimal via points indicated in Fig. 3.b with black crosses. From these via points, the B-spline interpolated trajectories at each joint $j$ are obtained up to their second derivative at each iteration of the optimization algorithm (see section III.B). The subregressors $\overline{\mathbf{W}}_{b i}^{D}$ used in the cost function of (18) are calculated for all $n$ samples by using the outputs of the B-spline function. Similarly, the constraints are satisfied for every sample $n$ of the trajectory. The second optimization process aims to ensure dynamic balance by modifying $\overline{\mathbf{q}}_{D B}\left(N_{J D B} \times N_{K}\right)$ as in the first optimization process. As mentioned previously, the total joint trajectory is composed of the two subsets of $\overline{\mathbf{q}}_{D}^{*}=\left[\overline{\mathbf{q}}_{D E}^{*}{ }^{T} \overline{\mathbf{q}}_{D B}^{*}{ }^{T}\right]^{T}$. The problem of finding the exciting motion between two consecutive postures $p$ and $p+1$ can be formulated as follows:

$$
\begin{aligned}
\text { Find } \overline{\mathbf{q}}_{D E}^{*} \in \quad & \min _{\overline{\mathbf{q}}_{D E} \in \mathbb{R}^{N_{J D E} \times N_{K}}} \sum_{i=1}^{N_{L D E}} M_{i} \operatorname{cond}\left(\overline{\mathbf{W}}_{b i}^{D}\right) \\
\text { s.t. } \quad & B S_{j}\left(0, q_{D E j}\right)=q_{S j}^{*}(p) \\
& B S_{j}\left(n T_{s}, q_{D E j}\right)=q_{S j}^{*}(p+1) \\
& \dot{B} S_{j}\left(0, q_{D E j}\right)=\dot{B} S_{j}\left(n T_{s}, q_{D E j}\right)=0 \\
& \ddot{B} S_{j}\left(0, q_{D E j}\right)=\ddot{B} S_{j}\left(n T_{s}, q_{D E j}\right)=0 \\
& q_{j}^{-} \leq B S_{j}\left(k T_{s}, q_{D E j}\right) \leq q_{j}^{+} \\
& \left|B_{j}\left(k T_{s}, q_{D E j}\right)\right| \leq \dot{q}_{j}^{+} \\
& \left|\bar{\Gamma}_{j}\right| \leq \Gamma_{j}^{+} \\
& \mu \bar{F}_{X, Y} \leq \bar{F}_{Z} \\
& 0 \leq \overline{\mathbf{d}}_{v e r t e x} \\
& \text { with } \quad j=1, . ., N_{J D E} \text { and } k=1, . .,(n-1)
\end{aligned}
$$

The first two optimization problems focused only on the head-arm-trunk segments, and considered 9 joints for the HOAP-3 and 12 joints for the human model. With $N_{K}=2$ via points per joint, it led to optimization problems of 18 and 24 variables each, respectively. The problem regarding the leg was of size for 12 both human and robot models. The specific 
constraints in (18) to be satisfied were relative to the maximal angular velocity, where $\dot{q}_{j}^{+}$is the maximal joint velocity and set to 1 and 4 rad.s ${ }^{-1}$ for the HOAP-3 and human model respectively and guaranteed that the friction forces act inside of the friction cone. For simplification, the Coulomb friction cone can be reduced to a squared base pyramid whose vertices are aligned with the axis of the frame associated with the contact surface [17]. Considering that the normal force has a constant sign and that foot contacts are always coplanar with the ground, it leads to simplified relationships between the normal $\left(F_{Z}\right)$ and tangential forces $\left(F_{X}, F_{Y}\right)$ under each foot:

$$
\mu \bar{F}_{X, Y} \leq \bar{F}_{Z} \quad \text { with } \quad \mu=0.5
$$

Once the exciting motions have been determined, a final optimization process uses the joints $\overline{\mathbf{q}}_{D B} *$ of the links that did not participate to the exciting motion to ensure balance. The exciting motions of the upper limbs might endanger dynamics balance, as shown in Fig. 6 in red.

By modifying the motion of the lower limbs it is possible to obtain a stable ZMP trajectory, as shown in green on Fig. 6. To do so, the deviation $J_{Z M P}=\left\|\mathbf{Z M} \mathbf{M}-\mathbf{Z} \bar{M} \mathbf{P}_{\text {Mid }}\right\|_{2}^{2}$ of the $\mathbf{Z} \bar{M} \mathbf{P}$ with respect to $\mathbf{Z} \bar{M} \mathbf{P}_{M i d}$, the center of the base of support, is minimized by solving the following optimization problem between two consecutive postures $p$ and $p+1$ :

$$
\begin{aligned}
\text { Find } \overline{\mathbf{q}}_{D B}^{*} \in & \min _{\overline{\mathbf{q}}_{D B} \in \mathbb{R}^{N_{J D B} \times N_{K}}} J_{Z M P} \\
\text { s.t. } \quad & B S_{j}\left(0, q_{D B j}\right)=q_{S j}^{*}(p) \\
& B S_{j}\left(n T_{s}, q_{D B j}\right)=q_{S j}^{*}(p+1) \\
& \dot{B S}\left(0, q_{D B j}\right)=\dot{B} S_{j}\left(n T_{s}, q_{D B j}\right)=0 \\
& \ddot{B} S_{j}\left(0, q_{D B j}\right)=\ddot{B} S_{j}\left(n T_{s}, q_{D B j}\right)=0 \\
& q_{j}^{-} \leq B S_{j}\left(k T_{s}, q_{D B j}\right) \leq q_{j}^{+} \\
& \left|\dot{B} S_{j}\left(k T_{s}, q_{D B j}\right)\right| \leq \dot{q}_{j}^{+} \\
& \mu \bar{F}_{X, Y} \leq \bar{F}_{Z} \\
& \left|\bar{\Gamma}_{j}\right| \leq \Gamma_{j}^{+} \\
& Z M P_{x, y}^{-} \leq Z \bar{M} P_{x, y} \leq Z M P_{x, y}^{+} \\
& 0 \leq \overline{\mathbf{d}}_{v e r t e x} \\
& \overline{\mathbf{P}}_{R F 1: 3_{x, y}}=\overline{\mathbf{P}}_{L F 1: 3_{x, y}}+\Delta \overline{\mathbf{P}}_{F} \\
& \text { with } \quad j=1, . ., N_{J D B} \text { and } k=1, . .,(n-1)
\end{aligned}
$$

Obviously the modification of the root-link's kinematic by the lower limbs movemement affects the excitation of the head-arm-trunk system. Fig. 5 shows the evolution of the criterion $J_{e x c}$ for the different postures for the HOAP-3 model. The two upper graphs $\left(J_{\text {excH } A T}\right)$ show the good global convergence of the criterion used to excite the head-arms-trunk system. As highlighted in this figure a few of these motions are poorly exciting, resulting in a slight increase in the criterion value. However, this sub-optimality is acceptable due to the large number of considered motions as successive optimization processes use the previous whole body trajectories to augment the regressor. For example, the criterion converges from 1702 to 13 over 28 posture transitions. A technical issue related
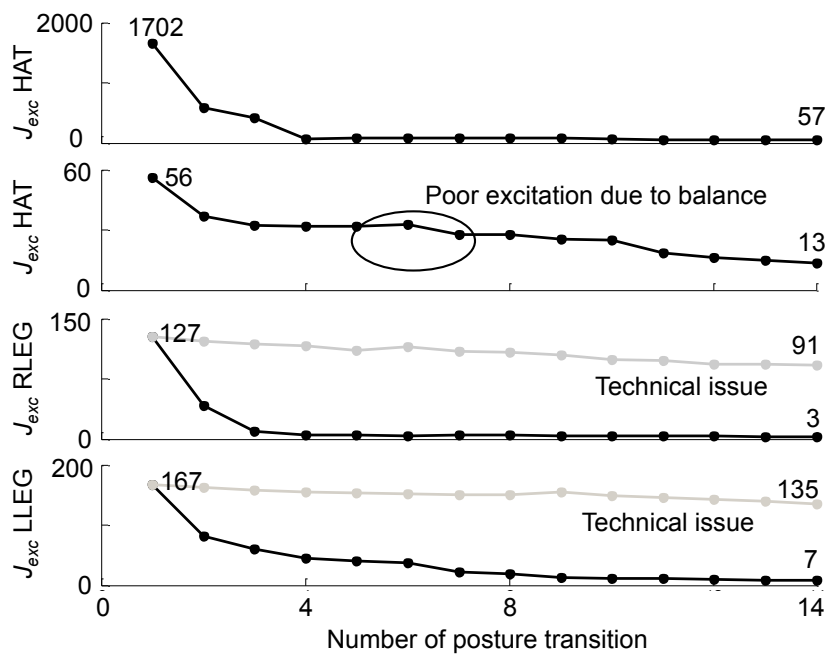

Fig. 5. Evolution of the excitation criterion (18) in function of the number of dynamic posture transition. Technical issues refers to a specific problem of our robot described in section IV.C.2.

to the ankle motors, detailed in section IV.C.2, forced us to constrain the minimization of the criterion $J_{\text {exc }}$ for the leg motions. The tolerance on the criterion was set to $5 \%$ of the previous value to obtain feasible postures for the real robot. This lead to trajectories that were less exciting than was initially expected. The bottom plots of Fig. 5 show the optimal evolution of criterion $J_{e x c}$ (in black) and its constrained version (in grey) used for the rest of the experimental validation. These non-linear constrained optimization processes were solved with the sequential quadratic programming (SQP) method using Matlab. The average time required to obtain each of the dynamic posture transition was of $8 \pm 4 \mathrm{~s}$.

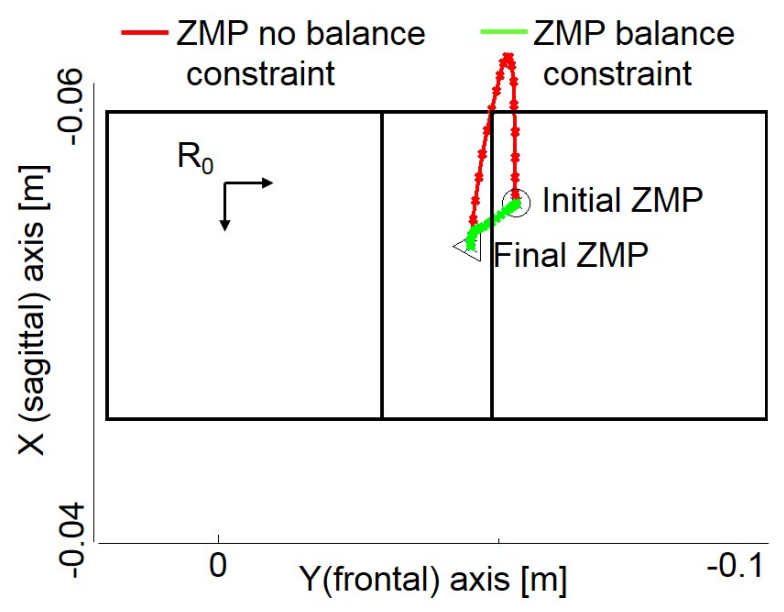

Fig. 6. Top view of the HOAP-3's simulated ZMP trajectories using only exciting motions, $\overline{\mathbf{q}}_{D E}^{*}$, of the upper limbs (red) and using lower limbs joints, $\overline{\mathbf{q}}_{D B}^{*}$, to maximize balance during posture transition (green). 


\section{EXPERIMENTAL VALIDATION}

\section{A. Human experimental setup}

One healthy and athletic female volunteer (age $=33$ years, weight $=65 \mathrm{~kg}$, height $=1.75 \mathrm{~m}$ ) participated in the study after signing an informed consent form. To familiarize the volunteer with the task, she was first asked to watch a slow motion video of all the generated optimal motions. Subsequently, the optimal trajectories of each body segment were superimposed onto a webcam's RGB video stream, as represented on Fig. 7.b, for visual biofeedback. Since the optimal motions $\overline{\mathbf{P}}_{\mathrm{D}}^{*}$ involve several segments at the same time, they might be difficult to replicate. To facilitate the identification procedure, during each optimal static posture $\mathbf{P}_{\mathbf{S}}{ }^{*}$, when the subject was not moving, the next motion $\overline{\mathbf{P}}_{\mathbf{D}}^{*} \in[p p+1]$ was shown. Finally, a visual instruction was given to the subject on when to move. The volunteer was asked to reproduce the projected optimal exciting motions, as closely as possible, while keeping her back straight. This identification phase lasted approximately $\left(\left(2\left(N_{p}-1\right)+5\left(N_{p}-1\right)\right) / 60=7\right.$ min $)$. Kinematic quantities were recorded using a stereophotogrammetric system (8 cameras, MX VICON). Fig. 7 presents the experimental setup used for the human validation experiment. Joint angles of the model displayed in Fig. 2 were calculated from a whole body set of 37 retro-reflective markers and a custom implementation of the classical multi-body optimization [30]. The markers were located at the anatomical landmarks specified by the plug-in-gait template (VICON). A force platform (AMTI BP-400600) was used to record the ground reaction forces and moments. Dynamometric and photogrammetric data were recorded at $100 \mathrm{~Hz}$ with respect to the same global frame in a synchronized fashion. The volunteer was asked to perform the identification process twice, the second time while wearing an additional load of $2.4 \mathrm{~kg}$ fixed to her left arm segment. This was done to assess the accuracy of the SIP identification process. The total mass of the subject was increased by $2.4 \mathrm{~kg}$ in the AT model when the subject was carrying the additional load. Once the SIP were estimated, the accuracy of the proposed method was also assessed over ten squats. The squats performed were very dynamic, with a large amplitude and high velocity, requiring the use of the arms to maintain balance.

\section{B. Human identification results}

Fig. 8 presents the comparison of the SIP obtained from AT and from the identification procedure with and without the additional mass placed at the left arm. Physical consistency constraints were respected and the least-square fitting of external wrench over the entire excitation dance was lower in the case of the identified model $(7.8 \pm 2.1 \mathrm{~N}$ and $4.3 \pm 1.9 \mathrm{~N} . \mathrm{m})$ than the AT parameters were used $(12 \pm 1.7 \mathrm{~N}$ and $9.67 \pm 3.65 \mathrm{~N} . \mathrm{m})$. As expected, most of the parameters were different between AT and the identified model. Some of the parameters, such as the masses and the COMs of the legs, display large differences. This result is almost impossible to validate but could be explained by the high athletic condition of the subject.

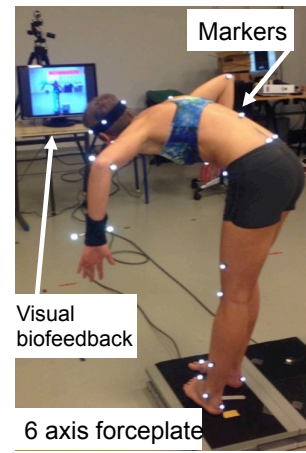

(a)

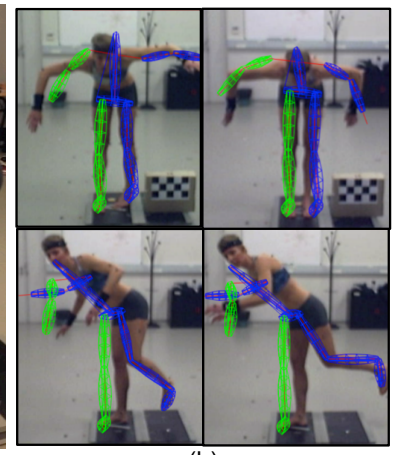

(b)
Fig. 7. Experimental setup used for the human validation (a). Views of the visual biofeedback used to display to the subject the optimal excitation trajectories (b).

However, one possible validation method was to compare the results of the identification performed with and without the additional mass. Most of the identified masses display a very similar value in both cases, the average mass difference between the two identified model was $0.25 \pm 0.17 \mathrm{~kg}$, with the largest difference observed at the trunk level $(0.42 \mathrm{~kg})$. However, the mass difference at the arm level was $1.9 \mathrm{~kg}$. The accuracy of the detection and of the estimation of the segment mass of our method was then $2.4-1.9=0.5 \mathrm{~kg}$. This result is consistent with previous literature studies. Ayusawa et al. [26] also estimated additional masses and reported an accuracy of $0.3 \mathrm{~kg}$. Our group, using a planar model, reported an accuracy of $0.5 \mathrm{~kg}$ [14].

1) Human cross validation results: Fig. 9 presents a crossvalidation result obtained during the squat exercise. The corresponding RMS and correlation coefficient values, calculated between the measured external wrench and their estimates using AT and identified models are given in Table II. The average RMS differences are much lower in the case of the identified model $(12.5 \mathrm{~N}$ and $3.2 \mathrm{~N} . \mathrm{m})$ than when using AT $(22.6 \mathrm{~N}$ and $7.9 \mathrm{~N} . \mathrm{m})$. The vertical force, subject to large accelerations, displays a much larger difference with an RMS error of 2.2 times larger for the AT model than for the identified one. Note that, from Fig. 9, this error is mainly displayed at the acceleration peaks and is not due to an offset on the subject weight. The same observation can be realized for the moment around the $\mathrm{X}$-axis. This will have a large influence if one would like to estimate the joint torque at the knee, for example, using only kinematic data [14]. The average correlation coefficient with the measured external wrench is also better with the identified model, $C C=0.73$, than with the AT model, $C C=0.64$.

\section{Robot experimental setup}

1) Robot motion validation: Prior to playing the actual motions onto the robot, a validation was performed using the Open Dynamics Engine in V-REP Simulator [29] and a custom CAD-valued model of HOAP-3. V-REP is useful since it embeds an efficient collision detection plugin that can be 


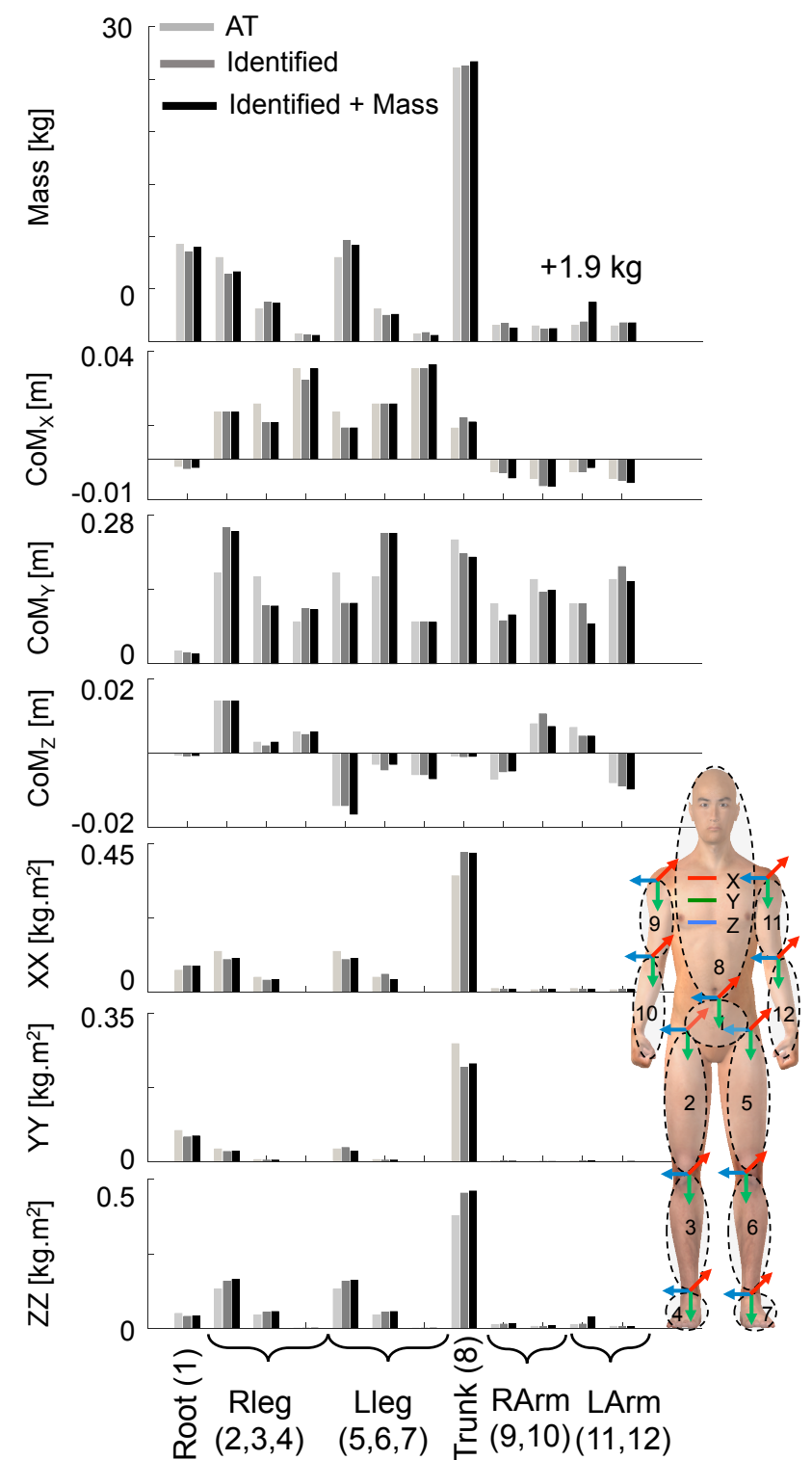

Fig. 8. Comparison of the AT and identified SIP.

used for the complex robot shapes. Fig. 10.a shows some of the 60 static postures in V-REP simulator.

2) Robot specific technical issue: The humanoid robot used in our experimentation was a HOAP-3 humanoid robot extensively used in various tasks the past seven years. During the experimental validation, several technical issues occurred. First of all, we used an external force platform that provides a better accuracy than the in-sole FSR sensor of the humanoid robot. Second, the pitch ankle motors, corresponding to a rotation in the sagittal plane, were gripped, limiting their range of motion. Finally, the flexibility on the actuation of the pitch ankle and the low friction values between the feet and the ground caused respectively overshoots and unbalanced movements. This made the identification of the inertia matrices of the legs links challenging. In order to cope with these

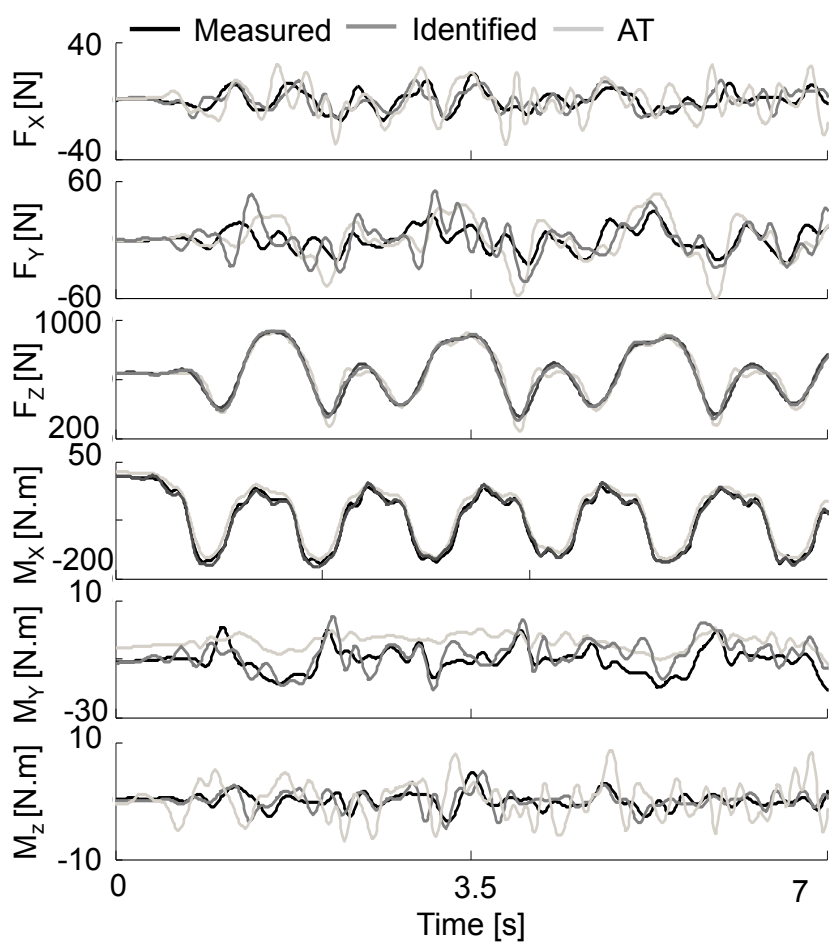

Fig. 9. Cross validation of the external forces and moments of force estimation realized during a squat exercise performed by a human subject.

TABLE II

COMPARISON OF THE MEASURED AND ESTIMATED EXTERNAL WRENCH AS ESTIMATED FROM AT/CAD DATA AND FROM IDENTIFICATION

\begin{tabular}{lcccccc} 
& Human & \multicolumn{5}{c}{ HOAP-3 } \\
\hline & & RMS & CC & & RMS & CC \\
\hline \hline$F_{X}[\mathrm{~N}]$ & Id & 5.8 & 0.54 & Id & 1.1 & 0.998 \\
& AT & 9.5 & 0.37 & CAD & 1.9 & 0.998 \\
$F_{Y}[\mathrm{~N}]$ & Id & 13.0 & 0.64 & Id & 0.5 & 0.997 \\
& AT & 16.4 & 0.60 & CAD & 0.6 & 0.997 \\
$F_{Z}[$ N] $]$ & Id & 18.9 & 0.98 & Id & 0.9 & 0.944 \\
& AT & 41.9 & 0.96 & CAD & 3.5 & 0.93 \\
$M_{X}[$ N.m] $]$ & Id & 4.9 & 0.99 & Id & 0.2 & 0.997 \\
& AT & 14.0 & 0.99 & CAD & 0.7 & 0.993 \\
$M_{Y}[$ N.m] & Id & 3.4 & 0.81 & Id & 0.7 & 0.998 \\
& AT & 6.6 & 0.79 & CAD & 0.8 & 0.994 \\
$M_{Z}[$ N.m] & Id & 1.5 & 0.43 & Id & 0.4 & 0.27 \\
& AT & 3.1 & 0.17 & CAD & 0.4 & 0.23
\end{tabular}

specific issues, the ankle pitch joints were constrained in the optimization procedure to a very limited range of motion around their zero position.

3) Robot data acquisition: As described in Fig. 10.b, the HOAP-3 robot was located on top of a force platform used to record the external wrench $(1000 \mathrm{~Hz}$, Accugait, AMTI). Seven retro-reflective markers were located on the trunk and feet links of HOAP-3. A stereophotogrammetric system $(100 \mathrm{~Hz}$, VICON Bonita) was used to collect marker trajectories and to estimate the waist position and orientation of the robot relative to the force platform system of reference in a synchronous fashion. Subsequently, the external ground reaction forces and moments were expressed in the root-link frame. The root- 


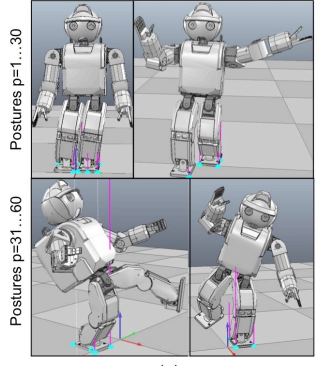

(a)

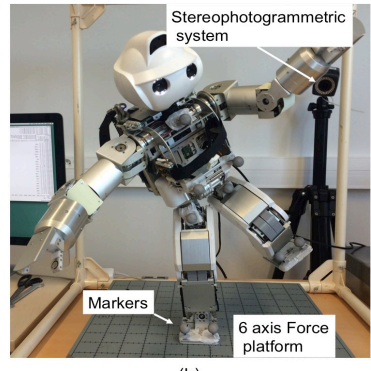

Fig. 10. View of the V-REP Simulator during double (postures 1 to 30) and single (postures 31 to 60) support postures (a). Experimental setup used for the identification of the HOAP-3 robot (b).

link identification can also be performed using the robot embedded force sensors and the robot geometrical model. The joint positions were recorded from robot encoders at 500 Hz. Optimal joint trajectories were tracked and reproduced onto the robot using the manufacturer PID controller at each joint. All collected data were processed using a $10 \mathrm{~Hz}$ cut-off frequency $5^{\text {th }}$ order low-pass, zero-phase filter.

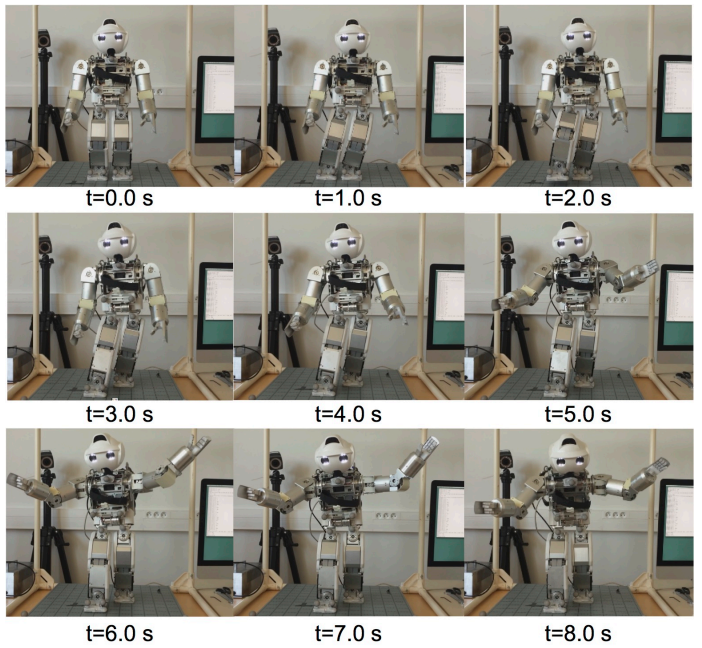

Fig. 11. Typical automatic repositioning from initial position (from 0 to $3 \mathrm{~s}$ ) and optimal exciting trajectory (the last $5 \mathrm{~s}$ ).

\section{Robot identification results}

The vector of BP was fully identified. However, from the identified parameters, only the ones with a relative standard deviation lower than $10 \%$ are deemed accurate enough for SIP identification. Indeed, a small humanoid robot implies small inertia parameter values. As such they are expected to have a much larger relative standard deviation and thus more difficult to identify [6]. From the prescribed optimal trajectories, 42 BP can be reliably identified. These parameters are almost all of the CoM BP and some of the main regrouped inertia components for the links corresponding to the head-arms-trunk system. As expected, the inertias of the lower legs and feet are hardly identifiable using the optimal trajectories for the legs as they were over-constrained due to the technical issues.
However, the method was able to successfully identify the CoM BP without needing to manual re-position of the robot, an improvement over previous studies [15]. Fig. 12 shows the comparison between CAD and identified links' masses, CoMs, and diagonal terms of the inertia matrices for the HOAP-3 robot. Physical consistency constraints were respected and the least-square fitting of the external wrench had a low RMS (less than $2 \mathrm{~N}$ and $1 \mathrm{~N} . \mathrm{m}$ ). As expected, the masses and several of the first moment of inertia were different from the CAD data. Most of the inertias did not play an important role in the robot dynamics and they were found to be very similar to the CAD data ones. This can be explained by the minimization of $\left\|\boldsymbol{\Phi}_{C A D}-\boldsymbol{\Phi}\right\|_{2}^{2}$ in (8). This shows that our method guarantees physical consistency, even when some parameters are poorly excited.

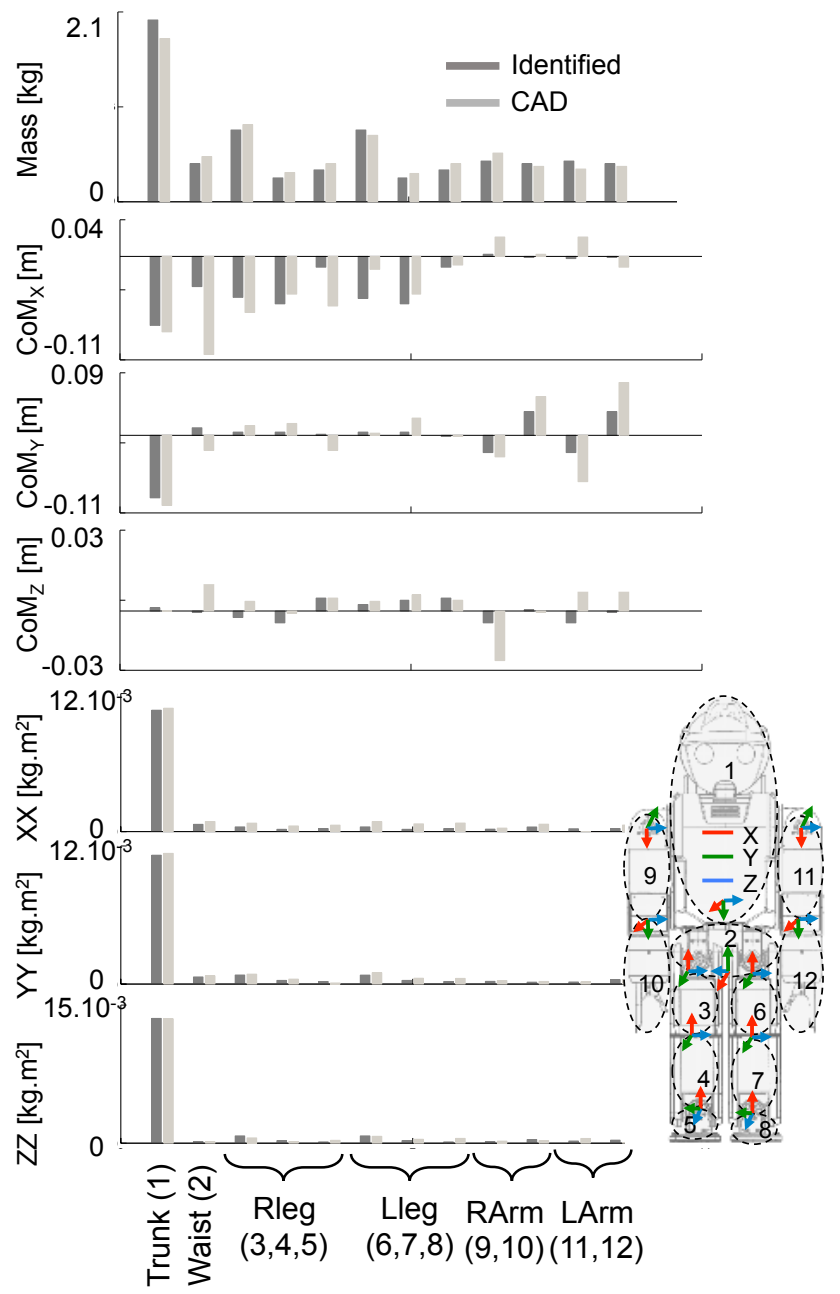

Fig. 12. Comparison of the CAD and identified SIP.

1) Robot cross validation results: A cross validation was performed with a motion that was not used during the identification process. A transition motion from the initial half sitting position to the first initial optimal pose, where the robot is in single support on the right foot, was retained. As it can be seen in Fig. 13, the identification process allowed for a 
better estimate of the external wrench than the CAD data. The corresponding RMS errors and correlation coefficients are summarized in Table II. A large difference is observable along the vertical force $F_{Z}$. This is due to the fact that the total mass of the robot was different than the one predicted by CAD. This difference in mass can be partially explained by the change or the removal of some of the robot's covers at the trunk and leg level, as well as the removal of cables used to connect the battery. The horizontal force and moment $\left(F_{X}, M_{X}\right)$ also displayed an improvement, where the RMS between measured and estimated quantities was reduced by a factor two. Neither the CAD nor the identified models were able to correctly predict the moment $M_{Z}$ around the vertical axis, but the amplitude of this signal is very low and below the accuracy of the force platform.

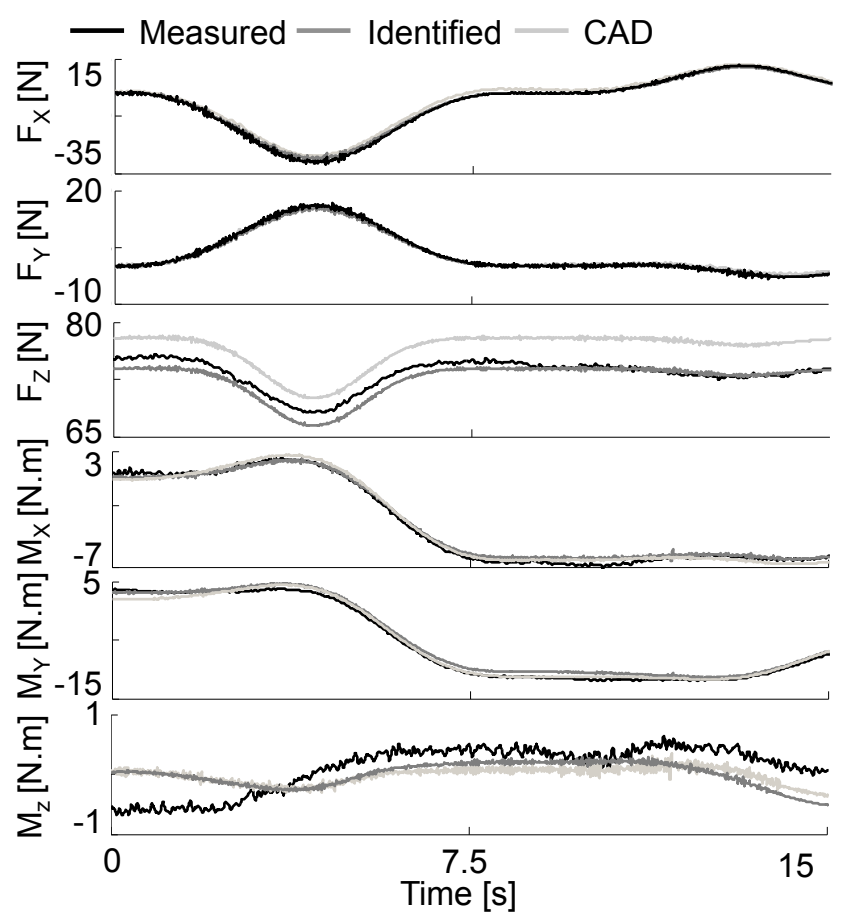

Fig. 13. Cross validation of the external forces and moments of force estimation during a HOAP-3 motion.

\section{CONCLUSION}

In this paper, a new method capable of generating continuous exciting motions for the identification of SIP of whole body 3D anthropomorphic structure has been proposed. It uses solely joint angles and contact force measurements expressed at the root-link level over a number of static and dynamic postures to identify the mass, CoM, and inertia matrix of each link. Optimal exciting motions were obtained by solving several constrained nonlinear optimization problems. The optimal static postures (14) required to identify CoMs were found first, then dynamic motions (18) used to identify inertias were determined. During these motions, the dynamic balance was handled by DoFs attached to links that were not directly used to generate the excitation. The models and the experimentations were based on both a human and a HOAP-3 humanoid robot. Cross-validations of the identified model using the estimate of the external wrench showed better accuracy improvements than when using an AT or CAD based model. For the human subject, the RMS errors was on average two times smaller than when using AT. Such differences will have a large influence on the estimate of the knee joint torque, which is a variable of interest in rehabilitation. The robot RMS errors were very small with magnitudes similar to the literature [26], [6]. However, in these previous studies only base parameters were identified. Uniquely, for a 3D whole body anthropomorphic structure, the proposed approach was able to detect and estimate an additional mass located on the subject arm with an accuracy of $0.5 \mathrm{~kg}$. To the best of our knowledge, this study is the first to propose a complete method to identify all SIP of an anthropometric structure using optimal exciting motions and taking into account the physiological and/or mechanical constraints. In some biomechanics applications, the identification of local joint dynamics and muscle parameters is of interest; however, these joint parameters will not have influence on the generalized external wrench that is used as input of the identification process. To identify these influences, different input data, such as electromyographic signal, and non-linear muscle models should be added in the identification process [31]. The execution time and accuracy of the whole optimization procedure was very reasonable (less than 15 minutes) and the optimization always converged when proper initial conditions were chosen. This means the framework can easily be generalized to other robots. The optimal motion can be generated for whole-body identification (i.e. for a new robot) or emphasis can be given to specific parts of the body (i.e. for a robot that has been repaired or modified, or a patient that is following a segment specific rehabilitation process) by tuning the weight of the regressor columns (12). The continuous aspect of the identification process is interesting for the humanoid robotics community who would benefit from having more realistic dynamical models of their robots. The current approach plays the optimal exciting motions in open-loop and thus requires a good first approximation of the robot parameters. However, it might be possible to develop a pseudo-online identification process handling dynamical balance and mechanical constraints in real-time. At least, the robot dynamical balance could be handled by a classical real-time ZMP controller [1] using the embedded force sensors. This will allow to extend the size of the base of support used in the optimization process allowing more dynamic motions. The criterion and the fast quadratic program presented in this study could be use to identify SIP and eventually regenerate optimal motions, depending on robot or human motion capture system specific sensor noise and measurement artifacts. This could be part of a routine calibration process by asking the robot or the human subject to perform a sort of calibration dance during a short clinical examination.

Technical issues described in section IV.C. 2 forced us to 
adapt our method to obtain better dynamic stability of the robot. However, this did not allow for the proper excitation of the robot leg inertias. In any case, the robot, in its current state, would not be able to generate motions influenced by leg inertias. This is very similar to the situation where pathological subjects suffer from muscular loss and/or reduced joint range of motion. The proposed approach could take into account specific subject limitations while generating the exciting motion. However, question of the ergonomy of the visual interface used to project the $3 \mathrm{D}$ complex motion to the subject will have to be addressed further. A visual bio-feedback similar to the ones used in dance video games with a Kinect sensor would pave the way for the development of a future tool in diagnostic decision-making [14].

\section{ACKNOWLEDGMENT}

The authors would like to acknowledge Dr. Christine Azevedo-Coste (INRIA, LIRMM, Montpellier, France) and Dr. Deborah Varoqui for their assistance in collecting motion capture data.

\section{REFERENCES}

[1] S. Kajita, F. Kanehiro, K. Kaneko, K. Yokoi, H. Hirukawa, "The 3D Linear Inverted Pendulum Mode: A simple modeling for a biped walking pattern generation", IEEE/RSJ Int. Conf. on Int. Robots and Systems, pp. 239-246, 2001.

[2] R. Dumas, L. Chèze, J. Verriest, "Adjustments to McConville et al. and Young et al. body segment inertial parameters", J. Biomech., vol. 40, pp. 543-553, 2007.

[3] G. Venture, K. Ayusawa, Y. Nakamura, "Motion capture based identification of human inertial parameters", IEEE/EMBS Int. Conf. on Eng. Med. and Bio., pp. 4575-4578, 2008.

[4] M. Mistry, S. Schaal, K. Yamane, "Inertial parameter estimation of floating base humanoid systems using partial force sensing", IEEE-RAS Int. Conf. on Humanoid Robots, pp. 492-497, 2009.

[5] K. Yamane, "Practical kinematic and dynamic calibration methods for force-controlled humanoid robots", IEEE-RAS Int. Conf. on Humanoid Robots, pp. 269-275, 2011

[6] K. Ayusawa, G. Venture, Y. Nakamura, 'Identifiability and Identification of Inertial Parameters Using the Underactuated Base-Link Dynamics for Legged Multibody Systems", Int. J. Robot. Res., 2013.

[7] S. Dubowsky, E. Papadopoulos, "The kinematics, dynamics, and control of free-flying and free-floating space robotic systems", IEEE Trans. on Robot. Autom., vol. 9, pp. 531-543, 1993.

[8] G. Venture, K. Ayusawa, Y. Nakamura, "A numerical method for choosing motions with optimal excitation properties for identification of biped dynamics - An application to human", IEEE/RSJ Int. Conf. on Int. Robots and Systems, pp. 1126-1131, 2009.

[9] K.J. Park, "Fourier-based optimal excitation trajectories for the identification of robots", Robotica, vol. 24, pp. 625-633, 2006.

[10] J. Swevers, C. Ganseman, D. Bilgin, J. De Schutter, H. Van Brussel, "Optimal robot excitation and identification", IEEE Trans. on Robot. Autom., vol. 13, no. 5, pp. 730-740, 1997.

[11] J. Jin and N. Gans, "Parameter identification for industrial robots with a fast and robust trajectory design approach", Rob. and Comp. Int. Manuf., vol. 31, pp. 21-29, 2015.

[12] W. Rackl, R. Lampariello, G. Hirzinger, "Robot Excitation Trajectories for Dynamic Parameter Estimation using Optimized B-Splines", IEEE/RSJ Int. Conf. on Int. Robots and Systems, pp. 2042-2047, 2012.

[13] C. Press and M. Gautier, "New criteria of exciting trajectories for robot identification", IEEE Int. Conf. on Robot. Autom., pp. 907-912, 1993.

[14] V. Bonnet and G. Venture, "Fast determination of the planar body segment inertial parameters using affordable sensors", IEEE Trans. Neural Syst. Rehabil. Eng., vol. 23, pp. 628-635, 2015.

[15] J. Baelemans, P.V. Zutven H. Nijmeijer, "Model parameter estimation of humanoid robots using static contact force measurements", IEEE Int. Symp. On safety, security, and rescue robotics, pp.1-6, 2013.
[16] J. Mayr, H. Gattringer "Static Inertial Parameter Identification for Humanoid Robots Using a Torque-Free Support", IEEE-RAS Int. Conf. on Humanoid Robots, pp. 99-104, 2014.

[17] T. Robert, J. Causse, G. Monnier, "Estimation of external contact loads using an inverse dynamics and optimization approach: General method and application to sit-to-stand maneuvers", J. Biomech., vol. 46, pp. 22202227, 2013.

[18] W. Khalil, E. Dombre, "Modeling, identification and control of robots", Eds. Herms Penton, London, United Kingdom, 2002.

[19] W. Khalil, D. Creusot ", SYMORO+: A system for the symbolic modeling of robots", Robotica, vol.15, pp.153-161, 1997

[20] Y. Fujimoto, S. Obata, A. Kawamura, "Robust biped walking with active interaction control between foot and ground", IEEE Int. Conf. on Robot. Autom., p. 2030-2035, 1998.

[21] M.B. Popovic, A. Goswami, H. Herr, "Ground reference points in legged locomotion: Definitions, biological trajectories and control implications", Int. J. Robot. Res., vol. 24, pp.1013-1032, 2005.

[22] Y. Xiang, J.S. Arora, K. Abdel-Malek, "Optimization-based prediction of asymmetric human gait", J. Biomech., vol. 44, pp-683-693, 2011.

[23] M. Gautier, W. Khalil, "On the identification of inertial parameters of robots", IEEE Int. Conf. on Robot. Autom., pp. 2264-2269, 1998.

[24] M. Gautier, "Numerical calculation of the base inertial parameters", $J$. of Robotic Systems, vol. 8, pp. 485-506, 1991.

[25] M. Gautier, G. Venture, "Identification of standard dynamic parameters of robots with positive definite inertia matrix", IEEE/RSJ Int. Conf. on Int. Robots and Systems, pp.5815-5820, 2013.

[26] K. Ayusawa, G.Venture, Y.Nakamura, "Real-time implementation of physically consistent identification of human body segments", IEEE Trans. on Robot. Autom., Shanghai, China, pp. 6282-6287, 2011.

[27] U. Zsolt, L. Lasdon, J.C. Plummer, F. Glover, J. Kelly, R. Marti, "Scatter search and local NLP Solvers: A Multistart framework for global optimization", INFORMS J. Comp., vol. 19, pp. 328-340, 2007.

[28] M. Gautier, W. Khalil, "Exciting trajectories for the identification of base inertial parameters of robots", Int. J. Robot. Res., vol. 11, no. 4, pp. 363-375, 1992

[29] E. Rohmer, S.P.N. Singh, M. Freese, "V-REP: a Versatile and Scalable Robot Simulation Framework", IEEE/RSJ Int. Conf. on Int. Robots and Systems, pp.1321-1326, 2013.

[30] T.W. Lu, J.J. O'Connor, "Bone position estimation from skin marker coordinates using global optimisation with joint constraints", J. Biomech., vol. 32, 129-134, 1999.

[31] M. Benoussaad, P. Poignet, M. Hayashibe, C. Azevedo-Coste, C. Fattal, D. Guiraud, "Experimental parameter identification of a multi-scale musculoskeletal model controlled by electrical stimulation: application to patients with spinal cord injury", Med Biol Eng Comput, vol. 51, 129134, 2013. 